\title{
Efficient Pre-Conditioned Descent Search Detector for Massive MU-
} MIMO

\section{Journal Article}

Author(s):

Zhang, Chuan; Jin, Jiejun; Xue, Ye; Tan, Xiaosi; Studer, Christoph (D); Zhang, Zaichen; You, Xiaohu

Publication date:

2020-05

Permanent link:

https://doi.org/10.3929/ethz-b-000452587

Rights / license:

In Copyright - Non-Commercial Use Permitted

Originally published in:

IEEE Transactions on Vehicular Technology 69(5), https://doi.org/10.1109/TVT.2020.2975878 


\title{
Efficient Pre-Conditioned Descent Search Detector for Massive MIMO
}

\author{
Chuan Zhang, Member, IEEE, Jiejun Jin, Student Member, IEEE, Ye Xue, Student Member, IEEE, \\ Zaichen Zhang, Senior Member, IEEE, Xiaohu You, Fellow, IEEE
}

\begin{abstract}
Being an enabling technique of the next generation wireless, massive multiple-input multiple-output (MIMO) can greatly enhance the spectral capacity and support much higher data rates. However, with hundreds of antennas, optimal massive MIMO detection suffers from prohibitive complexity. Thus, descent search (DS) algorithms have been employed because of their low-computation load and hardware-friendly features. However, the performance is not always guaranteed with varying antenna configurations or correlations. In this paper, a linear inequality constraint quadratic programming (LICQP) model is proposed first. The resulting constrained DS (CDS) detector outperforms the DS one. Furthermore, a universal pre-conditioner is proposed to improve the detector's convergence in imperfect conditions. Theoretical and numerical results indicate the preconditioned detector's advantage over the state-of-the-art (SOA) with $60 \%$ complexity reduction. Hardware implementation on Xilinx Virtex-7 shows that, compared with the SOA, our design achieves $1.75 \times$ throughput ( 35 Mbps in $128 \times 8$ i.i.d. channel) with similar hardware. Design framework proposed in this paper can be generalized to any other similar detectors.
\end{abstract}

Index Terms-Massive MIMO detection, constrained descent search, pre-conditioner, standard condition number, VLSI.

\section{INTRODUCTION}

$\mathbf{W}$ ITH the rapid growth of information industry, the volume of wireless data, number of intelligent devices, and speed of data transmission have been exploding in decades. The ITU IMT-2020 propulsion group has identified key features for the next generation mobile communication including: $100 \times$ higher energy efficiency, $5 \times$ higher spectrum efficiency, $10^{6}$ connections per square kilometer, and $1 \mathrm{Gbps}$ user experienced data rate.

In order to fulfill these requirements, [I] proposes five key techniques for future wireless communications, among which massive multiple-input multiple-output (MIMO) has captured great attentions. In a massive MIMO system, the base station (BS) serves a variety of users in the same frequency band with hundreds of antennas. Compared to small-scale MIMO, massive MIMO inherits its advantages and greatly surpasses it by providing more stable link connections, higher spectral efficiency, and more effective energy utilization. However, one of the problems coming along with massive MIMO is

C. Zhang, J. Jin, Z. Zhang, and X. You are with the National Mobile Communications Research Laboratory, Southeast University, China. Email: chzhang@seu.edu.cn.

Y. Xue was with the National Mobile Communications Research Laboratory, Southeast University, China. She is now with Electronic and Computer Engineering, Hong Kong University of Science and Technology, China.

C. Zhang and J. Jin contribute equally to this paper. (Corresponding author: Chuan Zhang.) the detection complexity: the optimal detection is NP-hard [2]. Though hardware capability has well developed in past decades, the implementation of optimal detector based on maximum likelihood (ML) or maximum a posteriori (MAP) is prohibitive for massive MIMO. Hence, sub-optimal detectors with low complexity are essential for practical applications.

Implementation-friendly detectors fall into two categories: 1) linear detectors and 2) non-linear detectors. Compared with non-linear ones, linear schemes such as zero forcing (ZF) [3] and minimum mean square error (MMSE) [4] require lower complexity to achieve reasonable performance. However, both of them need exact matrix inversion, and complexity of $\mathrm{QR}$ or Cholesky decomposition is $\mathcal{O}\left(U^{3}\right)$, where $U$ is the number of single-antenna users [5].

Inversion-free methods are then investigated. Approximate methods like Neumann series expansion (NSE) [6] calculate the inverse by finite expansion. However, when the number of NSE terms is greater than 2, its complexity returns to $\mathcal{O}\left(U^{3}\right)$. Another choice is the iterative linear detector such as descent search (DS), which iteratively searches towards the decline direction of the objected function. DS methods are hardwarefriendly, include steepest descent (SD) [ [Z], Barzilai-Bowein (BB) [8], conjugate gradient (CG) [9], and so on. In [10], a reconfigurable CG MIMO detector is proposed. In [II]], an SD detector for massive MIMO is introduced. However, there are still challenges:

1) Performance of DS detectors decreases drastically with the increase of transmitting antennas. How to improve the performance for massive MIMO is an issue.

2) DS detectors show weak convergence when applied to correlated channels. Thus, how to maintain performance advantages of DS detectors with varying antenna configurations or correlations is another issue.

\section{A. Contributions}

This paper devotes itself in proposing approaches to address the aforementioned issues. Our main contributions are:

- We propose a new detection model based on MMSE. Algorithms proposed with this model can be more precise and reliable than before.

- We propose a constrained DS (CDS) detector with rigorous proof. Performance improvement has been verified theoretically and numerically.

- We introduce a low-complexity pre-conditioner to improve the convergence and performance of existing preconditioners [11, 12]. Theoretical analysis of standard 
condition number ( $\mathrm{SCN}$ ) in the Hessian matrix is given. The resulting detector is called symmetric successive over relaxation DS (SSORDS) detector.

- We give the efficient hardware implementation for the proposed detector. This architecture can be generalized to all SSORDS detectors and any other similar detectors.

It is noted that this paper provides a new framework, applicable to any particular DS detector with pre-conditioner to cope with the specific scenario, no matter ideal or correlated, small or large-scale. Such frame work can produce existing detectors in [10-[13] and fit practical applications well.

\section{B. Notations}

In this paper, lower-case and upper-case boldface letters indicate column vector and matrix, respectively. The operations $(.)^{T},(\cdot)^{*},(\cdot)^{H}, \mathbb{E}\{\cdot\}$ and $\langle$,$\rangle stand for transpose,$ conjugate, conjugate transpose, expectation, and inner product, respectively. $\Re($.$) and \Im($.$) denote the real and imaginary parts$ of a vector. $\mathbf{I}_{N}$ is an $N \times N$ identity matrix and its $k$-th column is $\mathbf{I}(k)$. The entry in the $i$-th row and $j$-th column of $\mathbf{A}$ is $\mathbf{A}_{(i, j)}$; the $i$-th entry of $\mathbf{a}$ is $a_{i}$. The vector $\mathbf{a}$ in the $k$-th iteration is $\mathbf{a}^{(k)}$. For an $M$-dimensional square matrix $\mathbf{S}$, its eigenvalue is $\lambda_{i}(\mathbf{S})(i=1,2, \cdots, M)$, where $\lambda_{\max }(\mathbf{S})$ and $\lambda_{\min }(\mathbf{S})$ are the largest and smallest eigenvalues, respectively. $\mathbf{A} \leq \mathbf{B}$ in $\mathbb{R}^{M \times N}$ means $\mathbf{A}_{(i, j)} \leq \mathbf{B}_{(i, j)}$ for $i=1,2, \cdots, M$ and $j=1,2, \cdots, N$. The cardinality of a set $\mathcal{A}$ is $|\mathcal{A}|$.

\section{Paper Outline}

The rest of this paper is organized as follows. Section III gives preliminaries of massive MIMO. The CDS detector

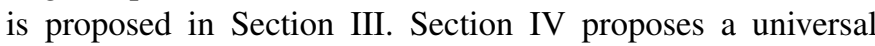
pre-conditioner in detail. Numerical results are discussed in Section D. FPGA implementation is presented in Section DI. Finally, Section $\nabla \mathbf{I}$ concludes the entire paper.

\section{PRELIMINARIES}

In this section, the massive MIMO system model, channel model, and detection model are given. The system model represents the physical scene of with mathematical equations. Correlation is introduced in our channel model. The ML detector is employed to set up the convex optimization model and the linear inequality constraint quadratic programming (LICQP) detection model is proposed.

\section{A. Uplink System Model}

In this paper, we consider a single cellular massive MIMO system with one BS equipped by $B$ antennas to serve $U$ singleantenna users at the transmitter side (TS). For a specific uplink case, the received symbol $\mathbf{y} \in \mathbb{C}^{B}$ at the BS in one symbol duration can be described as:

$$
\mathbf{y}=\sum_{i=1}^{U} \mathbf{h}_{i} s_{i}+\mathbf{n},
$$

where $s_{i}$ is the symbol from the $i$-th user at the TS, with power $\mathbb{E}\left\{\left\|s_{i}\right\|^{2}\right\}=1 . \mathbf{n}=\left[n_{1}, n_{2}, \cdots, n_{B}\right]^{T} \in \mathbb{C}^{B}$ is the additive white Gaussian noise (AWGN) and its elements are independent identical distributed (i.i.d.) with zero-mean and $\sigma^{2}$ variance. With $\mathbf{H}=\left[\mathbf{h}_{1}, \mathbf{h}_{2}, \cdots, \mathbf{h}_{U}\right]$, Eq. (四) becomes:

$$
\mathbf{y}=\mathbf{H s}+\mathbf{n},
$$

where $\mathbf{s}=\left[s_{1}, s_{2}, \cdots, s_{U}\right]^{T} \in \mathcal{O}^{U}$ is the transmitted vector, and $\mathcal{O}$ represents the QAM alphabets containing $2^{k}$ discrete signal points. Unlike flat Rayleigh fading channel, $\mathbf{H} \in \mathbb{C}^{B \times U}$ here is more complicated as discussed below.

\section{B. Channel Model}

In existing literature, the case with low load with 8 streams and ideal channel is widely discussed, whereas the case with medium/high load or correlated channel is not. However, such non-ideal condition exists in reality and significantly affects the channel characteristics, limiting the conventional detectors. To this end, the Kronecker model [14] is adopted here for a comprehensive analysis. Its mathematical description is:

$$
\mathbf{H}=\mathbf{R}_{B}^{1 / 2} \mathbf{T R}_{T}^{1 / 2},
$$

where $\mathbf{T} \in \mathbb{C}^{B \times U}$ is the complex standard Gaussian distributed channel with i.i.d. entries, i.e., $\mathbf{T}_{(i, j)} \sim \mathcal{C N}(0,1)$, with $i=1,2, \cdots, B$ and $j=1,2, \cdots, U \cdot \mathbf{R}_{B} \in \mathbb{C}^{B \times B}$ and $\mathbf{R}_{T} \in \mathbb{C}^{U \times U}$ are the correlation matrices of the BS and TS, respectively. The definitions are:

$$
\begin{aligned}
& \mathbf{R}_{B_{(i, k)}}=\left\{\begin{array}{cc}
\left(\zeta_{B} e^{j \phi}\right)^{k-i}, & i \leq k, \\
\mathbf{R}_{B_{(k, i)}}^{*}, & i>k ;
\end{array}\right. \\
& \mathbf{R}_{T_{(i, k)}}=\left\{\begin{array}{cc}
\left(\zeta_{T} e^{j \phi}\right)^{k-i}, & i \leq k, \\
\mathbf{R}_{T_{(k, i)}}^{*}, & i>k .
\end{array}\right.
\end{aligned}
$$

where $\zeta_{B}$ and $\zeta_{T}$ are coefficients which evaluate the correlations, and $\phi$ is the given phase. Eq. (B) is derived by assuming $\mathrm{BS}$ and TS are independent, since the BS and TS are far from each other.

According to [13], four scenarios are specified to further study the detection performance. Most researches only consider the ideal channel, where $\mathbf{R}_{B}^{1 / 2}$ and $\mathbf{R}_{T}^{1 / 2}$ degenerate to identity matrices $\mathbf{I}_{\mathbf{B}}$ and $\mathbf{I}_{\mathbf{U}}$, respectively. However, in this paper, since the antennas configuration in massive MIMO uplink is relatively dense, TS and BS are correlated. The correlation coefficients $\zeta_{B}$ and $\zeta_{T}$ are no longer 0 . Hence, $\mathbf{H}$ is in its complete form as Eq.s (4) and (5).

\section{LICQP Detection Model}

For massive MIMO, the ML detector [15] handles the detection problem in Eq. (2) by solving:

$$
\tilde{\mathbf{s}}^{\mathrm{ML}}=\underset{\mathbf{s} \in \mathcal{O}^{U}}{\arg \min }\|\mathbf{y}-\mathbf{H s}\|_{2}^{2} .
$$

However, the complexity makes it prohibitive for implementation. One way to reach the sub-optimal result with lower complexity is the BOX constraint optimization [16-18], which relaxes the constraint $\mathbf{s} \in \mathcal{O}^{U}$ to a convex hull around the constellation, i.e., $\mathbf{s} \in \mathcal{C}_{\mathcal{O}}^{U}\left(s_{i} \in \mathcal{C}_{\mathcal{O}}, i=1,2, \cdots, U\right)$, and $\mathcal{C}_{\mathcal{O}}$ 
is defined as in [19]:

$$
\left\{\sum_{i=1}^{|\mathcal{O}|} \theta_{i} s_{i} \mid s_{i} \in \mathcal{O}, \theta_{i} \geq 0, \theta_{i} \in \mathbb{R}, \forall i, \sum_{i=1}^{|\mathcal{O}|} \theta_{i}=1\right\} .
$$

As for the QAM discussed in this paper, $\mathcal{C}_{\mathcal{O}}=\left\{\left(x_{r}, x_{i}\right) \mid x_{r} \in\right.$ $\left.[-a, a], x_{i} \in[-a, a]\right\}$, and $a=\max _{a^{\prime} \in \mathcal{O}} \Re\left\{a^{\prime}\right\}$.

In fact, the BOX constraint $\mathbf{s} \in \mathcal{C}_{\mathcal{O}}^{U}$ can be regarded as a polytope in a two-dimensional space. Suppose $\overline{\mathbf{s}}$ is the real value decomposition (RVD) of $\mathbf{s}$, that is $\overline{\mathbf{s}}=\left[\begin{array}{ll}\Re(\mathbf{s})^{T} & \Im(\mathbf{s})^{T}\end{array}\right]^{T}$. Then, the constraint is in a linear form. Moreover, just like the MMSE, we can add a penalized item in the expression and acquire the LICQP [20] detection model:

$$
\begin{array}{ll}
\underset{\mathbf{s}}{\operatorname{minimize}} & \|\mathbf{y}-\mathbf{H s}\|_{2}^{2}+\sigma^{2}\|\mathbf{s}\|_{2}^{2}, \\
\text { subject to } & \mathbf{q} \overline{\mathbf{s}}^{T}-\mathbf{T} \leq \mathbf{0},
\end{array}
$$

where $\mathbf{q}=(1,-1)^{T}, \mathbf{T}=(a, a)^{T} \times \mathbf{e}_{2 U}^{T}$, and $\mathbf{e}_{2 U}$ is a $2 U \times 1$ vector whose elements are all 1 .

The proposed LICQP model is the basis of the designed methods below. Since the problem in Eq. (ID) is convex, it can be solved with numerical algorithms for convex optimization. More importantly, discussions in Section III show that methods under such model are much more effective than those with MMSE equalization.

\section{Constrained Descent Search Methods}

In this section, DS detector is introduced first, followed by the CDS detector. Advantages of CDS detector are identified theoretically and numerically. Challenges due to more user antennas and correlated channel are also pointed out.

\section{A. Feasibility of DS Detector}

According to the model in Eq. (पD), if we remove the constraint, the problem becomes an unconstrained minimization:

$$
\operatorname{minimize} \quad f(\mathbf{s})=\|\mathbf{y}-\mathbf{H s}\|_{2}^{2}+\sigma^{2}\|\mathbf{s}\|_{2}^{2},
$$

which can be solved by differentiating $f(\mathbf{s})$ :

$$
\tilde{\mathbf{s}}=\left(\mathbf{H}^{H} \mathbf{H}+\sigma^{2} \mathbf{I}_{\mathbf{U}}\right)^{-1} \mathbf{H}^{H} \mathbf{y} .
$$

This is the result of the MMSE equalizer as well. For linear functions, to avoid the high detection complexity, one possible choice is the iterative method like DS detector:

$$
\hat{\mathbf{s}}^{(i+1)}=\hat{\mathbf{s}}^{(i)}+\alpha^{(i)} \mathbf{d}^{(i)},
$$

where $\alpha^{(i)}$ and $\mathbf{d}^{(i)}$ are the step length and searching direction at iteration $i$, respectively. Since $\alpha^{(i)}$ and $\mathbf{d}^{(i)}$ always satisfy the criteria: $\alpha^{(i)}>0$ and $\nabla f\left(\hat{\mathbf{s}}^{(i)}\right)^{T} \mathbf{d}^{(i)}<0$, DS detector can guarantee the convergence by ensuring the decrease of the iteration result, i.e., $f\left(\hat{\mathbf{s}}^{(i+1)}\right)<f\left(\hat{\mathbf{s}}^{(i)}\right)$, in the convex area as long as $\hat{\mathbf{S}}^{(i)}$ is not the exact point.

For the proposed channel model, the Hessian matrix $\mathbf{A}=$ $\mathbf{H}^{H} \mathbf{H}+\sigma^{2} \mathbf{I}_{\mathbf{U}}$ is Hermitian positive definite (HPD). Hence, almost all variants of the DS detector can be used to solve Eq. (8). The key issue of DS detector is to determine $\alpha^{(i)}$ and $\mathbf{d}^{(i)}$ in Eq. (II)). For discussion convenience, three representative DS detectors are discussed, SD, BB and CG. The specific $\alpha^{(i)}$ and $\mathbf{d}^{(i)}$ for each detector are listed in Table $\mathbb{\text { I. }}$.

TABLE I

STEP LENGTH AND SEARCHING DIRECTION FOR DS DETECTORS.

\begin{tabular}{c|c|c}
\hline Algorithm & $\alpha^{(i)}$ & $\mathbf{d}^{(i)}$ \\
\hline SD [耳] & $\frac{\left\langle\tilde{\mathbf{y}}^{(i)}, \tilde{\mathbf{y}}^{(i)}\right\rangle}{\left\langle\mathbf{A} \tilde{\mathbf{y}}^{(i)}, \tilde{\mathbf{y}}^{(i)}\right\rangle}$ & $\tilde{\mathbf{y}}^{(i)}$ \\
BB [8] & $i=0:$ the same as SD & $\tilde{\mathbf{y}}^{(i)}$ \\
CG [9] & $i \geq 1: \frac{\left\langle\tilde{\mathbf{y}}^{(i-1)}, \tilde{\mathbf{y}}^{(i-1)}\right\rangle}{\left\langle\mathbf{A} \tilde{\mathbf{y}}^{(i-1)}, \tilde{\mathbf{y}}^{(i-1)}\right\rangle}$ & \\
\hline & $\frac{\left.\tilde{\mathbf{y}}^{(i)}, \tilde{\mathbf{y}}^{(i)}\right\rangle}{\left\langle\mathbf{A} \tilde{\mathbf{p}}^{(i)}, \tilde{\mathbf{p}}^{(i)}\right\rangle}$ & $\tilde{\mathbf{p}}^{(i)}$ \\
\hline
\end{tabular}

\section{B. Proposed CDS Detector}

The DS detector can be optimized by incorporating the LICQP model. To transform the detector to constrained version, projective operations [19] are introduced:

$$
\operatorname{proj}_{\mathcal{C}_{\mathcal{O}}}(w)= \begin{cases}w, & \text { if } w \in \mathcal{C}_{\mathcal{O}}, \\ \arg \min _{x \in \mathcal{C}_{\mathcal{O}}}|w-x|, & \text { if } w \notin \mathcal{C}_{\mathcal{O}},\end{cases}
$$

where $\mathcal{C}_{\mathcal{O}}$ is the convex hull of the modulation constellation.

The DS detector can be improved according to the projection rule. The constrained SD (CSD) detector in Algorithm II is given as an example and others can be revised accordingly.

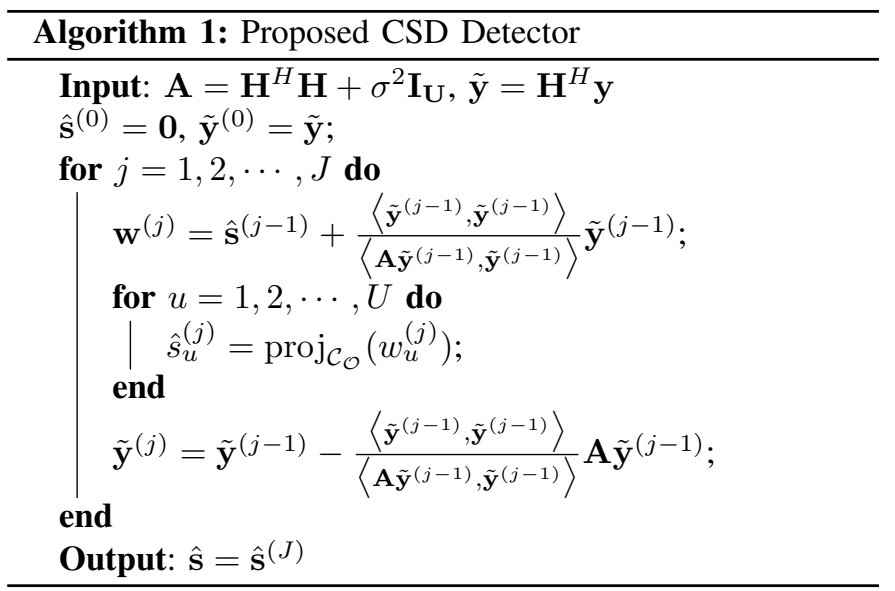

The convergence advantage of projective detector can be proved, by measuring the A-norm between the iterative result and the accurate one $\left.\mathbf{s}^{(*)}[\mathbf{8}, 2]\right]$. According to Proposition $\mathbb{Z}$, the efficiency of projection is guaranteed since the performance degradation of $\hat{\mathbf{s}}_{2}^{(j)}$ (with projection) is smaller than that of $\hat{\mathbf{s}}_{1}^{(j)}$ (no projection).

Proposition 1. Define $\|\mathbf{x}\|_{\mathbf{A}}=\sqrt{\langle\mathbf{A x}, \mathbf{x}\rangle}$, we have $\| \hat{\mathbf{s}}^{(*)}$ $\hat{\mathbf{s}}_{2}^{(j)}\left\|_{\mathbf{A}} \leq\right\| \hat{\mathbf{s}}^{(*)}-\hat{\mathbf{s}}_{1}^{(j)} \|_{\mathbf{A}}$.

Proof: See Appendix $\mathbb{B}-A$.

\section{Convergence Analysis of CDS Detector}

Proved in Section III-B, CDS detector can achieve superior performance over the DS one. In this subsection, numerical 


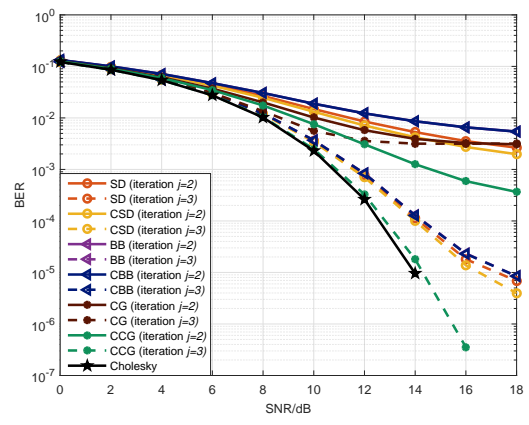

(a) $B=128, U=8, \zeta_{B}=0$, and $\zeta_{T}=0$.

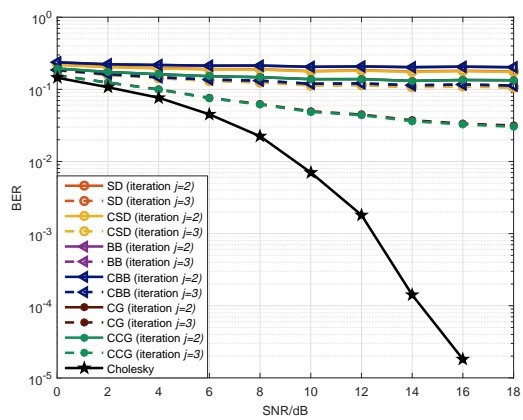

(d) $B=128, U=8, \zeta_{B}=0.2$, and $\zeta_{T}=0.4$.

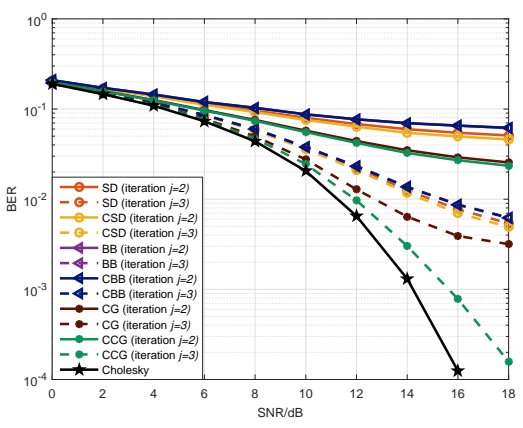

(b) $B=128, U=16, \zeta_{B}=0$, and $\zeta_{T}=0$.

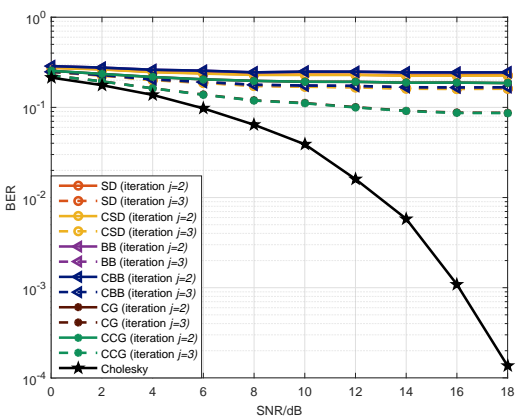

(e) $B=128, U=16, \zeta_{B}=0.2$, and $\zeta_{T}=0.4$.

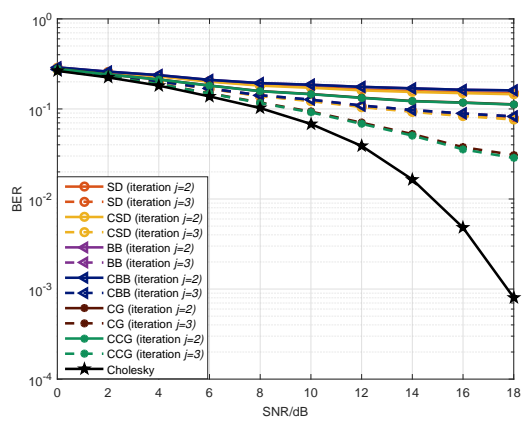

(c) $B=128, U=32, \zeta_{B}=0$, and $\zeta_{T}=0$.

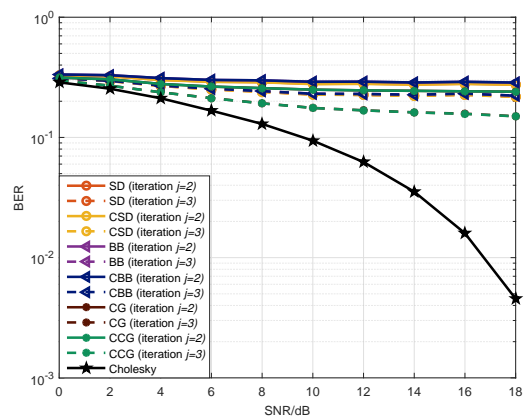

(f) $B=128, U=32, \zeta_{B}=0.2$, and $\zeta_{T}=0.4$.

Fig. 1. Performance of DS and CDS detectors with different antenna configurations and correlation factors.

results of both the CDS and DS detectors are given, where, the BS antenna number is 128 . Detector with Cholesky decomposition is employed as a baseline. According to Fig. W, it is noticed that regardless of the antenna configuration or channel correlation, constrained detectors outperform the other ones.

If the configuration is simple (Fig. 1(a)), the performance of CDS detector approximates the ideal result of Cholesky decomposition. However, with the growth of load (Fig.s [(a), ए(b), and (1(c)) or correlation (Fig.s ए(a) and ए(d)), all detectors suffer performance loss. In Fig. ए(t), where both the large load and correlation are considered, the performance degradation of all DS detectors becomes significant.

Indeed, the convergence can be mathematically analyzed. Firstly, we consider the DS detector. It is closely related to the $\mathrm{SCN} \kappa$ of Hessian matrix A [22], which is defined as:

$$
\kappa=\frac{\lambda_{\max }(\mathbf{A})}{\lambda_{\min }(\mathbf{A})} .
$$

The relationship between convergence rate of each DS detector and $\kappa$ is as below:

- SD [2T]:

$$
\left\|\mathbf{s}^{(*)}-\hat{\mathbf{s}}^{(j)}\right\|_{\mathbf{A}} \leq\left(\frac{\kappa-1}{\kappa+1}\right)^{j}\left\|\mathbf{s}^{(*)}-\hat{\mathbf{s}}^{(0)}\right\|_{\mathbf{A}}
$$

- BB $[8]$ :

$$
\left\|\mathbf{s}^{(*)}-\hat{\mathbf{s}}^{(j)}\right\|_{2} \leq(\kappa-1)^{j}\left\|\mathbf{s}^{(*)}-\hat{\mathbf{s}}^{(0)}\right\|_{2},
$$

- CG [23]:

$$
\left\|\hat{\mathbf{s}}^{(*)}-\hat{\mathbf{s}}^{(j)}\right\|_{\mathbf{A}} \leq 2\left(\frac{\sqrt{\kappa}-1}{\sqrt{\kappa}+1}\right)^{j}\left\|\hat{\mathbf{s}}^{(*)}-\hat{\mathbf{s}}^{(0)}\right\|_{\mathbf{A}}
$$

As a result, it can be inferred from Eq.s ([12), ([13), and (14) that if $\kappa$ is closer to 1 , the convergence will be faster. It has been proved in [24] that for matrix $\mathbf{A}_{p}=\mathbf{H}^{H} \mathbf{H}$, the increase of $U$ with a fixed $B$ will enlarge the probability of being ill-conditioned (with a large $\mathrm{SCN}$ ). The introduction of correlation under the same condition will deteriorate the result as well. We analyze $\mathbf{A}$ here with eigenvalue decomposition:

$$
\mathbf{A}=\mathbf{U}\left(\boldsymbol{\Lambda}_{p}+\sigma^{2} \mathbf{I}_{\mathbf{U}}\right) \mathbf{U}^{H},
$$

where $\mathbf{U}$ is a unitary matrix and $\boldsymbol{\Lambda}_{p}$ is a diagonal matrix whose elements equal the eigenvalues of $\mathbf{A}_{p}$. The relationship between the eigenvalues of $\mathbf{A}$ and those of $\mathbf{A}_{p}$ is:

$$
\lambda_{i}(\mathbf{A})=\lambda_{i}\left(\mathbf{A}_{p}\right)+\sigma^{2} .
$$

Since $\sigma^{2}$ can be regarded as a constant under certain circumstances, the difference of the eigenvalues of $\mathbf{A}$ and $\mathbf{A}_{p}$ is a constant, which means that the above conclusion of $\mathbf{A}_{p}$ can also be applied to $\mathbf{A}$. That is, the load increase and channel correlation will lead to a larger SCN of $\mathbf{A}$ and contribute to the convergence loss.

Comparison of A's SCN under different scenarios is in Fig. 口, and validates the above discussion. Here, $B$ is 128 , SNR is $5 \mathrm{~dB}$, but the number of user streams changes. Since the SCN obtained in simulations is a random variable, its mean is used to indicate the performance. When the user number 


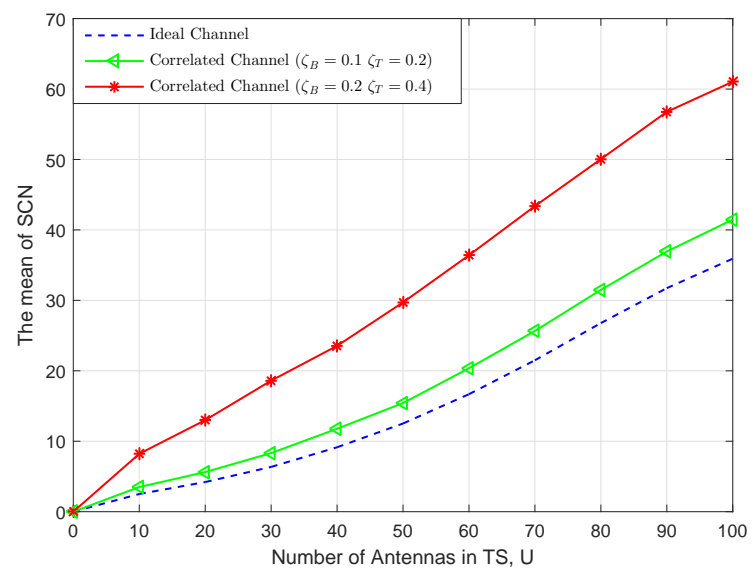

Fig. 2. SCN of matrix A with $B=128$ and $\mathrm{SNR}=5 \mathrm{~dB}$.

increases, all three curves increase. Meanwhile, the SCN in a fully correlated channel (red) is always greater than those in the less correlated one (green) and the uncorrelated one (blue). This difference leads to the performance degradation of DS and CDS detectors in Fig.s ए(c), ए(d), ए(e), and ए(t). To this end, the pre-conditioner is introduced below.

\section{Proposed Universal Pre-Conditioner}

In this section, motivations of the universal symmetric successive over relaxation (SSOR) pre-conditioner is discussed. Details of the per-conditioner are given.

\section{A. Motivations of Introducing Pre-Conditioner}

According to Section III-C, DS detector is not as robust as the direct detector. Although DS detector is low-complexity and implementation-friendly, its convergence remains an issue. Here, pre-conditioner is introduced as a solution.

Generally, pre-conditioning is a process, which transforms the original problem into an easier one with explicit and implicit operations. For example, scaling all numbers in a linear system to normalize the diagonal elements [12] is pre-conditioning. The general expression for linear preconditioning is:

$$
P_{\mathbf{M}}(f(\mathbf{x}))=\mathbf{M} f(\mathbf{x}),
$$

where $\mathbf{M}$ is called the linear pre-conditioner.

The aim of pre-conditioning is to lower the SCN $\kappa$ of $\mathbf{A}$. With the pre-conditioner, the Hessian matrix becomes MA. According to Eq. (II), the optimal value of $\kappa$ is the smallest one, indicating that the unit matrix owns the best SCN. As a result, if $\mathbf{M}=\mathbf{A}^{-1}$, the convergence can be maximized. However, inversion of large-scale matrix will bring prohibitive complexity. To better leverage complexity and performance, several pre-conditioners have been proposed. The split preconditioner (SP) in [I2] is simple but not feasible in correlated channel. The incomplete Cholesky (IC) decomposition based pre-conditioner [II] can boost the convergence with acceptable cost. However, it is not stable in complicated scenarios either.

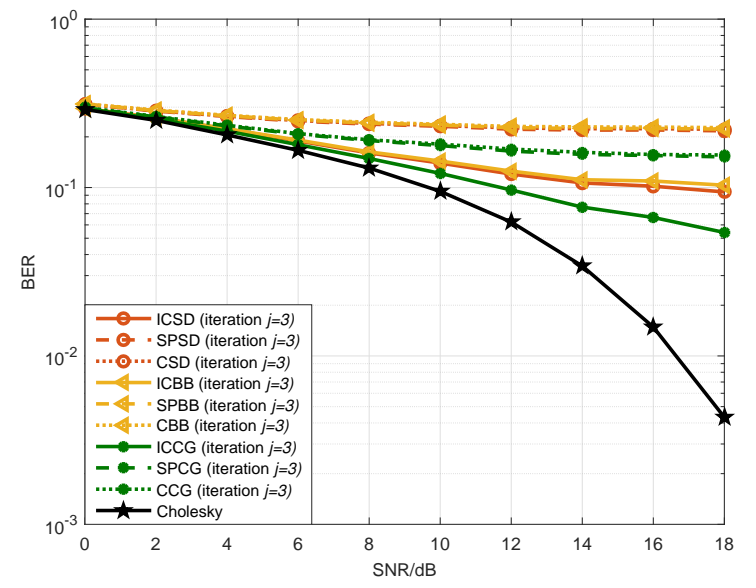

Fig. 3. Performance of SP and IC based pre-conditioner with $B=128, U=$ $32, \zeta_{B}=0.2$ and $\zeta_{T}=0.4$.

According to Fig. 及, when user load and channel correlation are high, detector with either pre-conditioner only shows similar performance as those without pre-conditioner. Hence, it is necessary to design proper pre-conditioner which performs well in various scenarios while keeping low complexity.

\section{B. Proposed SSOR Pre-Conditioner}

The pre-conditioner is expected to reduce $\mathrm{SCN}$ with low complexity. To this end, SSOR pre-conditioner is proposed. For an HPD matrix A, its split matrix after SSOR [25] is:

$$
\mathbf{M}=\frac{\omega}{2-\omega}\left(\frac{1}{\omega} \mathbf{D}-\mathbf{C}_{\mathbf{L}}\right) \mathbf{D}^{-1}\left(\frac{1}{\omega} \mathbf{D}-\mathbf{C}_{\mathbf{L}}{ }^{H}\right)=\mathbf{L} \mathbf{L}^{H},
$$

where $\mathbf{L}=\frac{\left(\mathbf{D}-\omega \mathbf{C}_{\mathbf{L}}\right) \mathbf{D}^{-\frac{1}{2}}}{\sqrt{\omega(2-\omega)}}, \mathbf{D}$ and $\mathbf{C}_{\mathbf{L}}$ are the diagonal and strictly lower triangle parts of $\mathbf{A}$. As long as $\mathbf{A}$ is positive definite and $0<\omega<2, \mathbf{M}$ is positive definite. Though the expression of $\mathbf{M}$ is complicated with an undetermined coefficient, we can take $\omega=1$ and achieve satisfactory performance. Then $\mathbf{L}$ is simplified as:

$$
\mathbf{L}=\mathbf{C}_{\mathbf{L}}{ }^{\prime} \mathbf{D}^{-\frac{1}{2}},
$$

where $\mathbf{C}_{\mathbf{L}}{ }^{\prime}$ is the lower triangle part of $\mathbf{A}$.

It is noted that even if $\mathbf{A}$ and $\mathbf{M}$ are symmetric, $\mathbf{M}^{-\mathbf{1}} \mathbf{A}$ may not be symmetric and not suitable to be solved by the DS method. Preprocessing is required before using the preconditioner. There are two possible methods:

1) Replace $\mathbf{A}$, $\hat{\mathbf{s}}$, and $\tilde{\mathbf{y}}$ with $\mathbf{A}^{\prime}=\mathbf{L}^{-1} \mathbf{A} \mathbf{L}^{-H}, \hat{\mathbf{s}}^{\prime}=\mathbf{L}^{H} \hat{\mathbf{s}}$, and $\tilde{\mathbf{y}}^{\prime}=\mathbf{L}^{-1} \tilde{\mathbf{y}}$, respectively. Then $\mathbf{A} \hat{\mathbf{s}}=\tilde{\mathbf{y}}$ becomes $\mathbf{L}^{-1} \mathbf{A} \mathbf{L}^{-H} \mathbf{L}^{H} \hat{\mathbf{s}}=\mathbf{L}^{-1} \tilde{\mathbf{y}}$. Since $\mathbf{L}^{-1} \mathbf{A} \mathbf{L}^{-H}$ is symmetric, it is suitable to compute with $\mathbf{A}^{\prime}, \hat{\mathbf{s}}^{\prime}$, and $\hat{\mathbf{y}}^{\prime}$ with CDS method to attain the pre-conditioner.

2) Alternatively, similar as the derivation of DS method, Proposition $\square$ can be obtained:

Proposition 2. If $\mathbf{A}$ and $\mathbf{M}$ are both $U \times U$ HPD matrices and $\tilde{\mathbf{y}} \in \mathbb{C}^{U}$, we have

$$
\mathbf{M}^{-1} \mathbf{A} \mathbf{s}^{*}=\mathbf{M}^{-1} \tilde{\mathbf{y}} \Leftrightarrow \hat{\varphi}\left(\mathbf{s}^{*}\right)=\min \hat{\varphi}(\mathbf{s}),
$$


where $\hat{\varphi}(\mathbf{s})=\frac{1}{2}\left\langle\mathbf{M}^{-1} \mathbf{A} \mathbf{s}, \mathbf{s}\right\rangle_{\mathbf{M}}-\left\langle\mathbf{M}^{-1} \tilde{\mathbf{y}}, \mathbf{s}\right\rangle_{\mathbf{M}}$ and $\langle\mathbf{x}, \mathbf{y}\rangle_{\mathbf{M}} \equiv\langle\mathbf{M x}, \mathbf{y}\rangle \equiv\langle\mathbf{x}, \mathbf{M y}\rangle$ is the $\mathbf{M}$-inner product of $\mathbf{x}$ and $\mathbf{y}$.

Hence, by replacing the inner products in the DS method with the M-inner products, the pre-conditioning can be properly applied.

Both methods are feasible and unified. For convenience, here the algorithm is given in the form of the latter one. As this pre-conditioner is applicable for all DS detectors, the universal pre-conditioned detector is called SSORDS detector. The particular algorithm of each DS detector is in Appendix 因, all of which can be derived from Algorithm D:

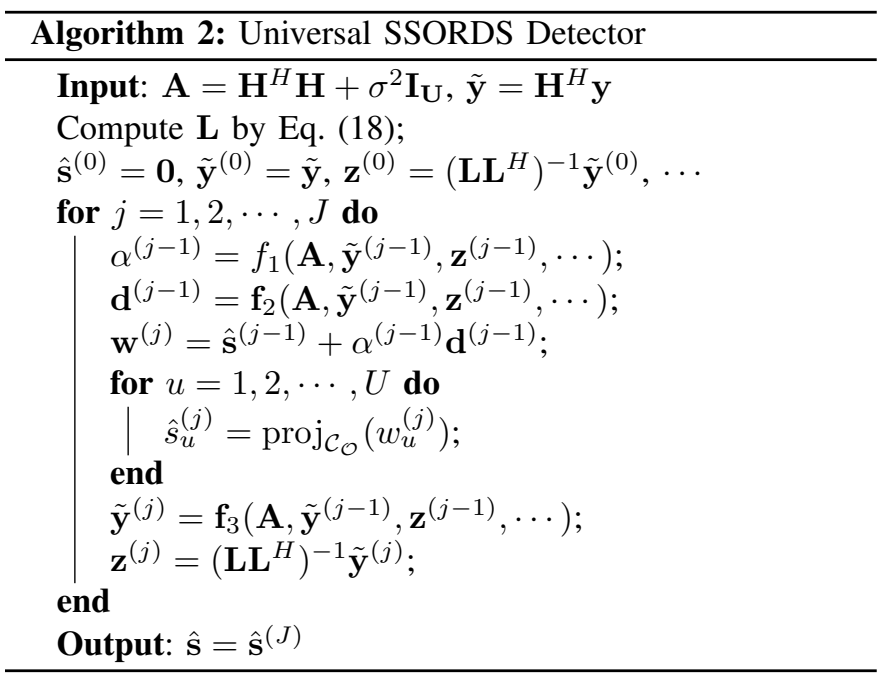

\section{Convergence Analysis of SSOR Pre-Conditioner}

SSOR pre-conditioner is to optimize the SCN of matrix A. It is proved to reduce SCN to its square root value, and is very effective for ill-conditioned matrix owing to complex configuration or correlated channel. The proof is in Appendix B-B. Numerical results in Fig. $\mathbb{A}$ illustrate the improvements.

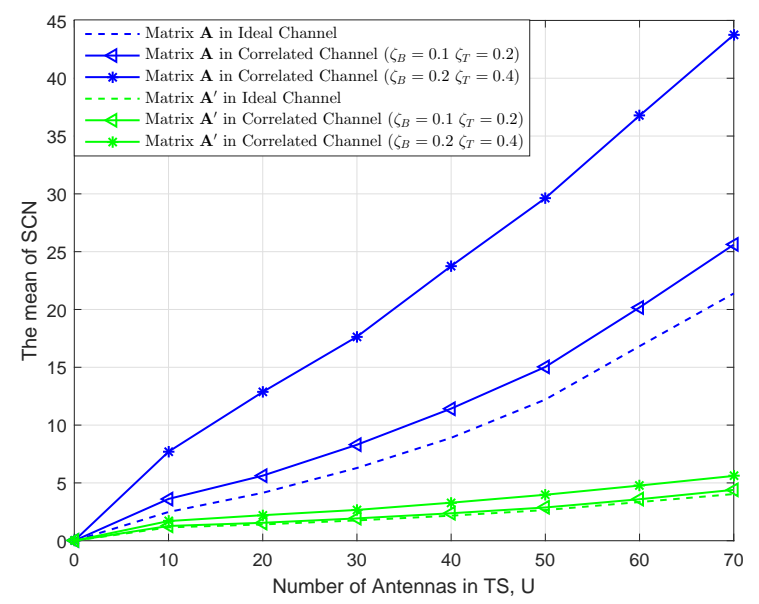

Fig. 4. SCN comparison between matrix $\mathbf{A}$ and $\mathbf{A}^{\prime}$ with $B=128$ and $\mathrm{SNR}=5 \mathrm{~dB}$.

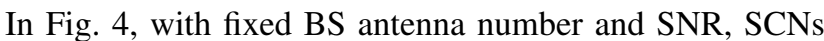
with such pre-conditioner roughly decrease to the square root values. In contrast, the previous pre-conditioned methods like SP and IC based algorithms can not achieve the same level as SSOR. Advanteges over existing pre-conditioners such as SP and IC are illustrated in Fig. [].

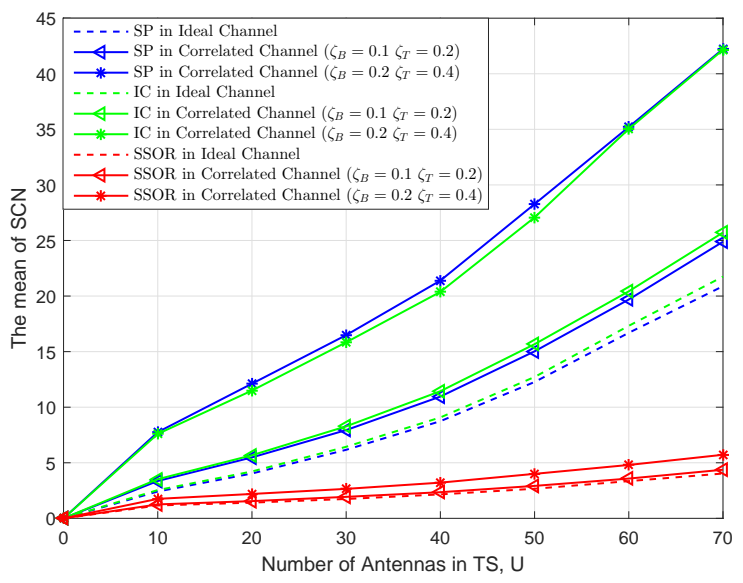

Fig. 5. SCN comparison of matrix $\mathbf{A}^{\prime}$ using SP, IC, and SSOR with $B=128$ and $\mathrm{SNR}=5 \mathrm{~dB}$.

\section{Complexity Analysis of SSOR Pre-Conditioner}

Though pre-conditioner introduces extra computational costs, it is acceptable compared to the ideal Cholesky decomposition based algorithms. The complexity is measured in terms of the number of complex-valued multiplications. Here SSORSD detector is used as a representative for the proposed SSORDS detectors.

1) Compute L: In Eq. (18), the square root and its reciprocal are stored in the look-up-table (LUT). Thus, the expression of $\mathbf{L}$ only includes the multiplication of a $U \times U$ lower triangle matrix with a diagonal one, and the complexity is $((U+1) U) / 2$.

2) Compute $\mathbf{z}^{(0)}: \mathbf{z}^{(0)}$ is calculated in two steps: $\mathbf{u}^{(0)}=$ $\mathbf{L}^{-1} \tilde{\mathbf{y}}^{(0)}$ and $\mathbf{z}^{(0)}=\mathbf{L}^{-H} \mathbf{u}^{(0)}$. Thus the total complexity includes 2 multiplications of a $U \times U$ lower triangle matrix with a $U \times 1$ vector, which is $(U+1) U$.

3) Compute $\hat{\mathbf{s}}^{(j)}: \hat{\mathbf{s}}^{(j)}$ is obtained from $\mathbf{w}^{(j)}$ by a projection without multiplication. So the complexity of computing $\hat{\mathbf{s}}^{(j)}$ is the same as that of $\mathbf{w}^{(j)}$. This step includes 2 inner products between two $U \times 1$ vectors, a multiplication of a $U \times U$ matrix with a $U \times 1$ vector, and a scalar multiplication with a $U \times 1$ vector. The complexity is $\left(U^{2}+3 U\right)$.

4) Compute $\tilde{\mathbf{y}}^{(j)}$ : Since $\frac{\left(\tilde{\mathbf{y}}^{(j-1)} \cdot \mathbf{z}^{(j-1)}\right)}{\left(\mathbf{A} \tilde{\mathbf{z}}^{(j-1)} \cdot \tilde{\mathbf{z}}^{(j-1)}\right)}$ and $\mathbf{A} \tilde{\mathbf{z}}^{(j-1)}$ have already been calculated in 3), the complexity is $U$.

5) Compute $\mathbf{z}^{(j)}$ : The complexity of this step is similar as that of computing $\mathbf{z}^{(0)}$, which equals $(U+1) U$.

Therefore, the overall complexity of the SSORSD detector is $\left(\left(2 k+\frac{3}{2}\right) U^{2}+\left(5 k+\frac{3}{2}\right) U\right)$. 


\section{Numerical Results}

In this section, numerical results regarding BER performance and computational complexity are given to demonstrate the advantages of the proposed detector.

\section{A. BER Performance}

The main disadvantage of CDS detector and existing preconditioned ones is the lack of stability. However, SSORDS detector is a good solution, shown in Fig.s 6 and $\mathrm{Z}$.

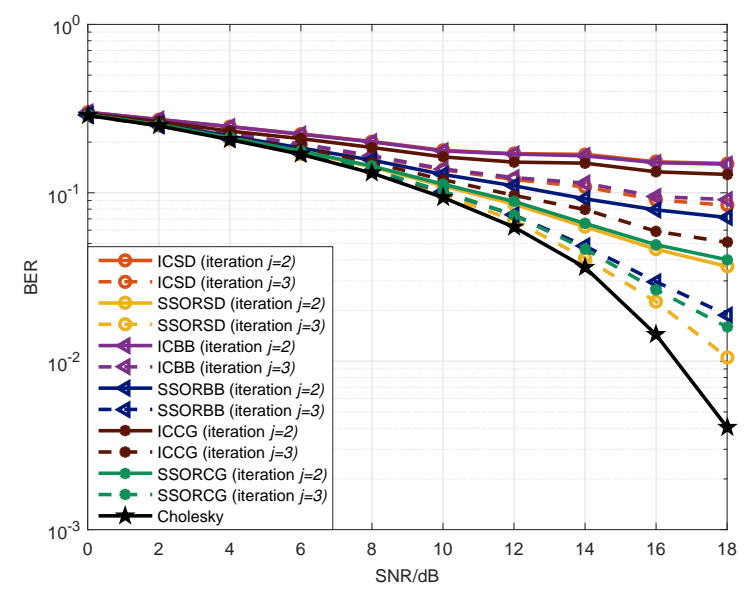

Fig. 7. Performance comparison between ICDS and SSORDS methods with $B=128, U=32, \zeta_{B}=0.2$ and $\zeta_{T}=0.4$.

In Fig. 6, when the channel is ideal, SSORDS detector with only 2 iterations can achieve similar convergence as the Cholesky one regardless of the user number. Even if the correlation in the BS and TS is considered, the results are satisfactory. Unlike the unsatisfactory performance in Fig. [(f), SSORDS detector in Fig. [6(f)] still shows good convergence. SSORDS detector outperforms all the other ones. For example, compared to ICDS detector in Fig. Z, SSORDS detector shows $2 \mathrm{~dB}$ performance gain even with one fewer iteration.

\section{B. Computational Complexity}

The computational complexity of SSORDS detector is given in Section IV-D. Here, it is compared with SOA detectors. Details are listed in Table II. CSD detector has the lowest complexity due to no extra operations. The complexity of SSORSD detector is slightly higher than that of SPSD detector, but lower than that of ICSD detector.

In Fig. 8, 40\% complexity reduction from ICSD detector is achieved when $U=88$ and over $60 \%$ when $U=100$. With the analysis in Section $\nabla-A$, the proposed SSORDS detector can achieve a good balance of performance and complexity. Its hardware implementation is given below.

\section{HARDWARE IMPLEMENTATION}

For better understanding, the hardware implementation of SSORSD detector is discussed as an example. The universal implementation for any SSORDS detector is then suggested. For comparison convenience, all multiplications are assumed to be complex-valued.
TABLE II

COMPUTATIONAL COMPLEXITY COMPARISON OF VARIOUS METHODS.

\begin{tabular}{c|c}
\hline Algorithm & Computational Complexity \\
\hline Cholesky Inversion & $\frac{5}{6} U^{3}+\frac{3}{4} U^{2}+\frac{4}{3} U$ \\
\hline CSD & $k\left(U^{2}+4 U\right)$ \\
SPSD & $2 k U^{2}+(5 k+1) U$ \\
ICSD [II] & $\frac{\left(U^{2}-2 S\right)^{3 / 2}-\left(U^{2}-2 S\right)^{1 / 2}}{6}+$ \\
& $(2 k+1) U^{2}+(5 k+1) U-(2 k+2) S$ \\
\hline Proposed SSORSD & $\left(2 k+\frac{3}{2}\right) U^{2}+\left(5 k+\frac{3}{2}\right) U$ \\
\hline
\end{tabular}

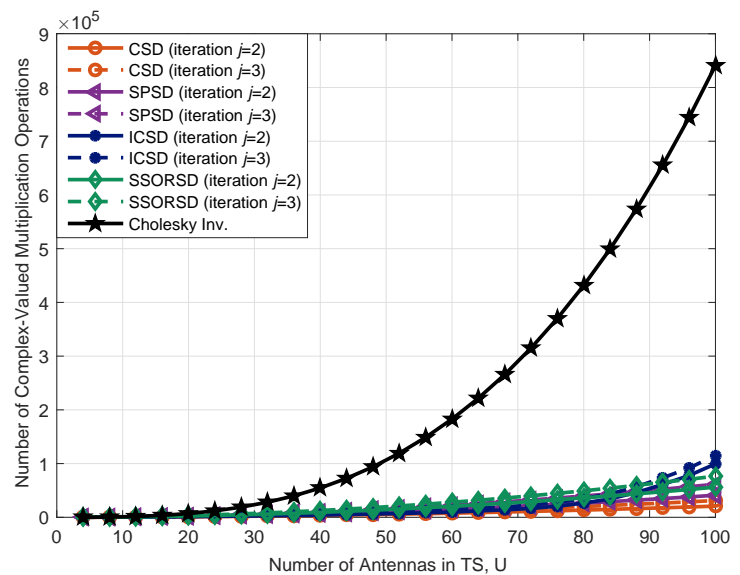

Fig. 8. Complexity comparison with $B=128$ and $\mathrm{SNR}=5 \mathrm{~dB}$.

\section{A. Hardware Architecture of SSORSD Detector}

According to Algorithm [3, SSORSD detector includes three parts: Preprocessing Unit, Iterative Unit, and Output Unit (Fig. Q). Iterative Unit runs the core function, i.e. the iterative SD method. Preprocessing Unit prepares A, $\tilde{\mathbf{y}}$, and the preconditioner $\mathbf{L}$. Output Unit calculates $\hat{\mathbf{s}}^{(j)}$ by projecting $\mathbf{w}^{(j)}$.

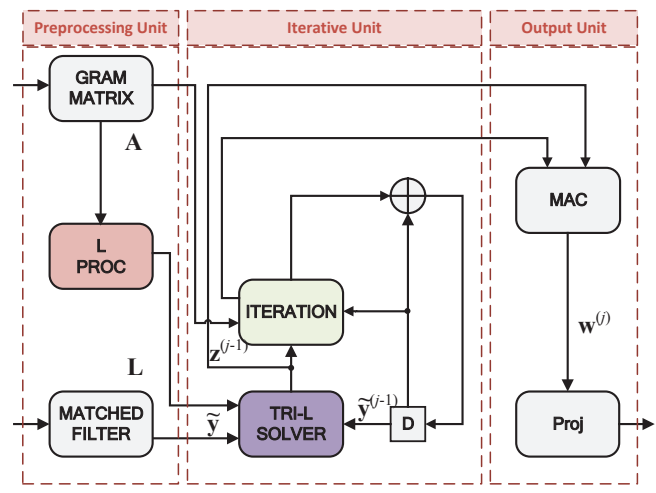

Fig. 9. Block diagram of SSORSD detector.

1) Data-Flow Graph Analysis: Data-flow graph (DFG) in Fig. [10 reveals the dependance of nodes in Fig. Q. Analysis on iteration bound and critical path [26] is as follows.

As for iteration bound, there are three loops in the DFG:

$$
l_{1}=\text { Node } 6 \rightarrow \text { Node } 6,
$$




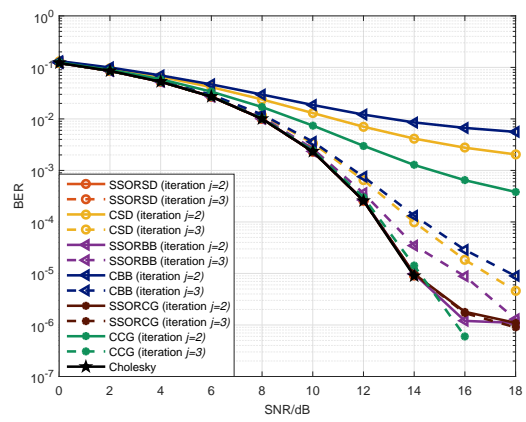

(a) $B=128, U=8, \zeta_{B}=0, \zeta_{T}=0$

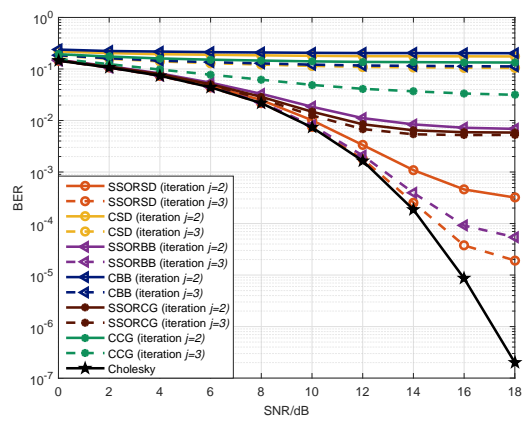

(d) $B=128, U=8, \zeta_{B}=0.2$, and $\zeta_{T}=0.4$.

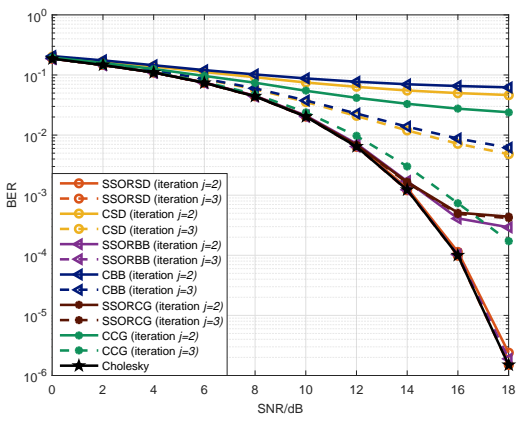

(b) $B=128, U=16, \zeta_{B}=0$, and $\zeta_{T}=0$.

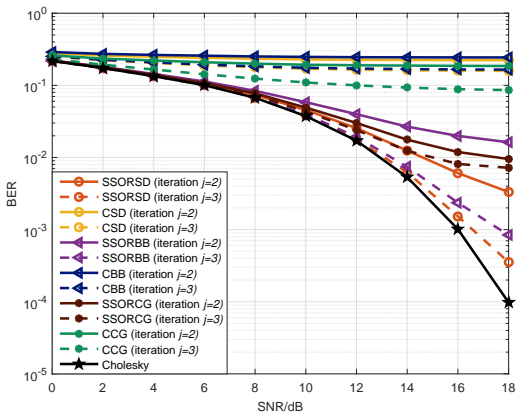

(e) $B=128, U=16, \zeta_{B}=0.2$, and $\zeta_{T}=0.4$.

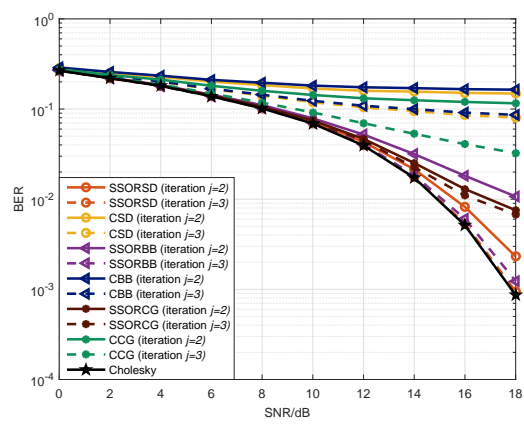

(c) $B=128, U=32, \zeta_{B}=0$, and $\zeta_{T}=0$

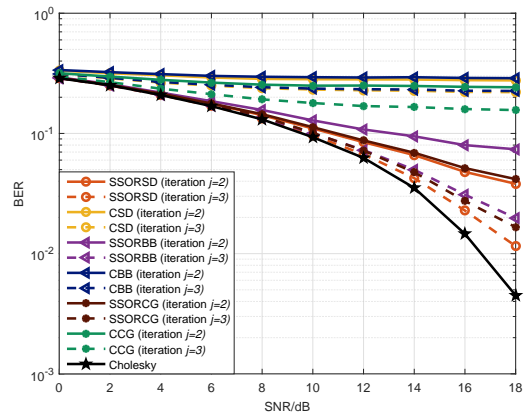

(f) $B=128, U=32, \zeta_{B}=0.2$, and $\zeta_{T}=0.4$.

Fig. 6. Performance comparison between CDS and SSORDS detectors with different antenna configurations and correlation factors.

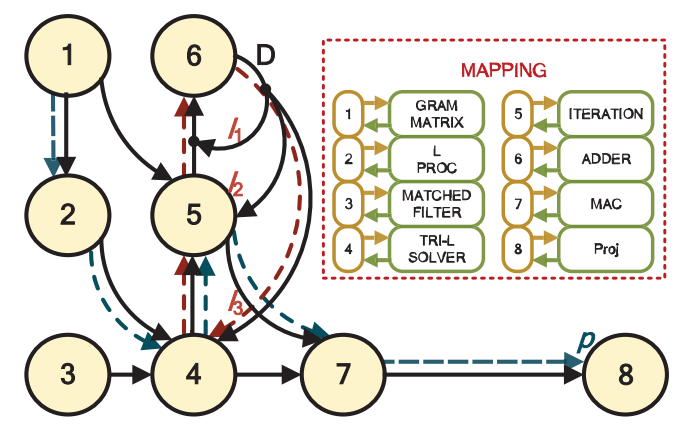

Fig. 10. DFG of SSORSD detector.

$$
\begin{aligned}
& l_{2}=\text { Node } 6 \rightarrow \text { Node } 5 \rightarrow \text { Node } 6, \\
& l_{3}=\text { Node } 6 \rightarrow \text { Node } 4 \rightarrow \text { Node } 5 \rightarrow \text { Node } 6 .
\end{aligned}
$$

Iteration bound $T_{\infty}=\max \left\{T_{l_{1}}, T_{l_{2}}, T_{l_{3}}\right\}=T_{l_{3}}=T_{6}+T_{5}+$ $T_{4}$, where $T_{l_{i}}$ is the length of loop $l_{i}$ and $T_{i}$ is the operation time of node $i$.

The critical path $p=$ Node $1 \rightarrow$ Node $2 \rightarrow$ Node $4 \rightarrow$ Node $5 \rightarrow$ Node $7 \rightarrow$ Node 8 . It also reveals the dependance of $N o d e 7$ upon $l_{2}$ and $l_{3}$.

2) Preprocessing Unit: MATCHED FILTER module for $\tilde{\mathbf{y}}$ is made up of $U$ processing elements (PEs). With multiplyaccumulate operation, each PE is associated with one row of $\mathbf{H}^{H}$ and one column of $\mathbf{H}$ or $\mathbf{y}$.

Similar as [6], GRAM MATRIX module for $\mathbf{A}$ is composed of a lower triangle systolic array including $(U+1) U / 2$ PEs [27]. The diagonal PEs are slightly different since they

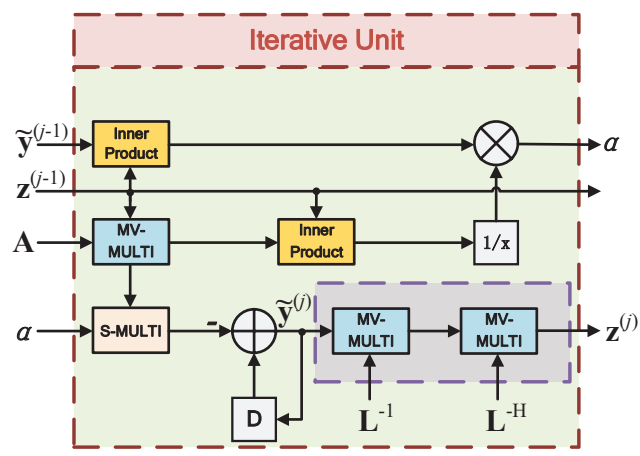

Fig. 11. Detailed architecture of Iteration Unit.

have to add noise $\sigma^{2}$. The multiplier used here and below contains 4 real-multipliers and 2 real-adders.

L PROC module is designed for the pre-conditioner $\mathbf{L}$. According to Eq. (18)), an LUT can be used to obtain $\mathbf{D}^{-\frac{1}{2}}$. Then $\mathbf{L}$ can be calculated through a multiplier array.

3) Iterative Unit: The iteration bound of this unit (Fig. III) is $T_{6}+T_{5}+T_{4}$, which is decided by Node6 (ADDER module), Node5 (ITERATION module), and Node4 (TRI-L SOLVER module).

For TRI-L SOLVER module, $\mathbf{L}^{-1}$ is acquired first with a special systolic array in Fig. [12 [28]. Considering the $k$ th column of $\mathbf{L}^{-1}$ as the solution of $\mathbf{L x}=\mathbf{I}(k)$, it takes $\left(2 U^{2}-U\right)$ cycles to compute the inversion. Then TRI-L SOLVER module solves the trigonometric equation in two steps: $\mathbf{u}^{(j-1)}=\mathbf{L}^{-1} \tilde{\mathbf{y}}^{(j-1)}$ and $\mathbf{z}^{(j-1)}=\mathbf{L}^{-H} \mathbf{u}^{(j-1)}$. These 
operations are based on matrix-vector multiplications and can reuse the preceding modules.

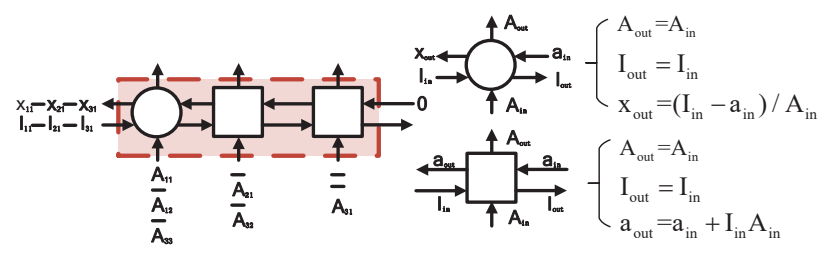

Fig. 12. Systolic Array which computes the inverse $\mathbf{L}^{-1}$.

Though ITERATION module looks complicated, there are only a few types of operations involved: matrix-vector product, vector inner product, vector scaling, reciprocal and vector addition (substraction). Since iterative operations of all DS detectors are similar, this unit can be generalized to other DS detectors. Details of the universal design is introduced in Section VI-B].

4) Output Unit: Multiply-accumulate operations help to compute $\mathbf{w}^{(j)}$. Proj module employs comparators and selectors for the projective function to output $\hat{\mathbf{s}}^{(j)}$.

5) Timing Analysis: GRAM MATRIX module and MATCHED FILTER module need $(B+2 U-1)$ and $(B+$ $U-1$ ) clock cycles, respectively. L PROC module works after GRAM MATRIX module. To obtain $\mathbf{L}^{-1}$ for TRI-L SOLVER module, $\left(2 U^{2}-U\right)$ clocks are required. For each iteration, it takes $(U+1)$ clocks to compute $\mathbf{z}^{(j)}$. Iterative Unit calculates $\mathbf{w}^{(j)}$ and $\mathbf{y}^{(j)}$ together in $3 U$ clocks, since they share common components. Finally, $\hat{\mathbf{s}}^{(j)}$ can be computed through Proj module. The overall timing analysis is displayed in Fig. [3].

6) FPGA Implementation: In order to show the efficiency of our design, implementation on Xilinx Virtex-7 XC7VX690T FPGA is given and compared with SOA. We use fixed-point arithmetic in our design: the quantization length of input and output equals 16 , and that of multipliers is 32 . The LUT has 256 addresses and 8-bit output.

In Fig. 14, the fix-point performance of SSORSD detector is compared with the SOA. It shows little difference from the float-point one. Moreover, they both outperform the SOA detectors even with one fewer iteration.

Implementation comparison with SSORSD detector, GS detector [6], adaptive IC based APSD detector [13], and CGLS detector [10] is listed in Table [W]. All of them are carried out on the same FPGA using 64-QAM for an $128 \times 8$ antenna configuration. It is noted that SSORSD detector achieves higher throughput than the SOA. Furthermore, it outperforms CGLS detector by $1.75 \times$ throughput while showing better convergence as in Section $\mathbb{Z}$. Its hardware efficiency in terms of Throughput/LUTs and Throughput/FFs is also better than the SOA. Therefore, the implemented detector shows better balance of detection performance and hardware efficiency.

\section{B. Universal Architecture of SSORDS Detector}

Since all SSORDS detectors share the same preprocessing unit (computing pre-conditioner, $\mathbf{A}$, and $\tilde{\mathbf{y}}$ ), output process $\left(\mathbf{w}^{(j)}=\hat{\mathbf{s}}^{(j-1)}+\alpha^{(j-1)} \mathbf{d}^{(j-1)}\right)$, projective function, and

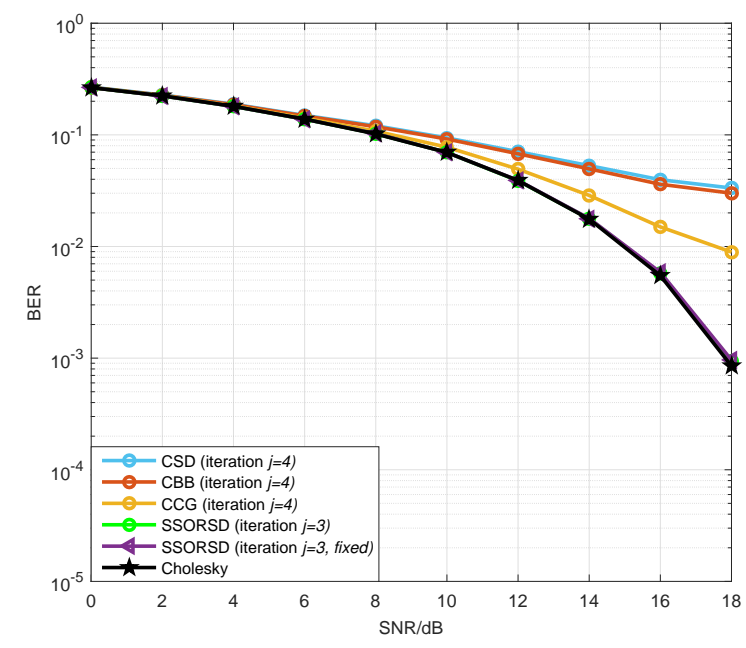

Fig. 14. Fix-point performance of SSORSD detector with $B=128$ and $U=32$ in i.i.d. channel.

auxiliary operations, a universal architecture can be designed. In this architecture, the Preprocessing Unit and Output Unit remain unchanged. The Iterative Unit has the same modules. Due to the change of number and operation order, switches and registers are required to slightly adjust the unit. The entire architecture is shown in Fig. [5. The Preprocessing Unit is omitted here for convenience as it can be found in Fig. Q 9 .

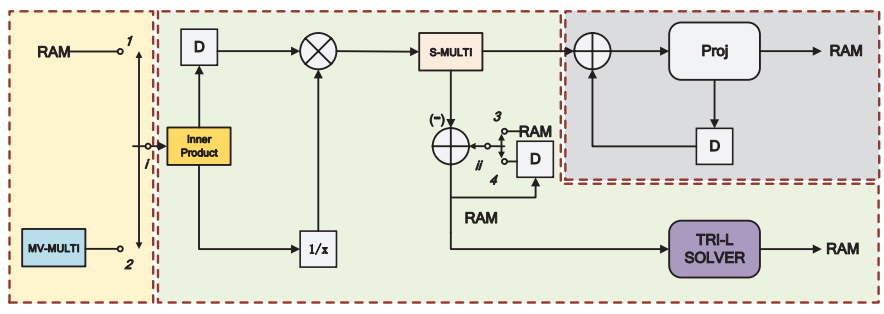

Fig. 15. Universal architecture of the proposed SSORDS detector.

In Fig. [5, S-MULTI module calculates the scale-vector product with multiplier array. The Inner Product module can reuse these multipliers and a U-to- 1 adder tree [10]. MVMULTI module, which is designed for matrix-vector product, can adopt the architecture of MATCHED FILTER module. The result of such multiplication can also be thought as a column of inner products and be computed with Inner Product modules. Thus, we may fold this module to reduce the hardware cost. High-throughput reciprocal calculation can be implemented with existing FPGA unit [29]. Since the rest modules in Fig. [55 have been discussed, their details are omitted here.

For a specific SSORDS detector, this universal architecture can be combined with the detailed algorithm. There are two switches in Fig. [5]. The iterative operations of SSORSD detector and SSORBB detector are similar: Switch $i$ chooses 1 or 2 and Switch ii keeps with 4 . Since SSORCG detector has one more variable, 3 needs to be considered.

This architecture satisfies all SSORDS detectors and achieve decent performance with acceptable complexity. We can choose the proper detector according to the specific needs 


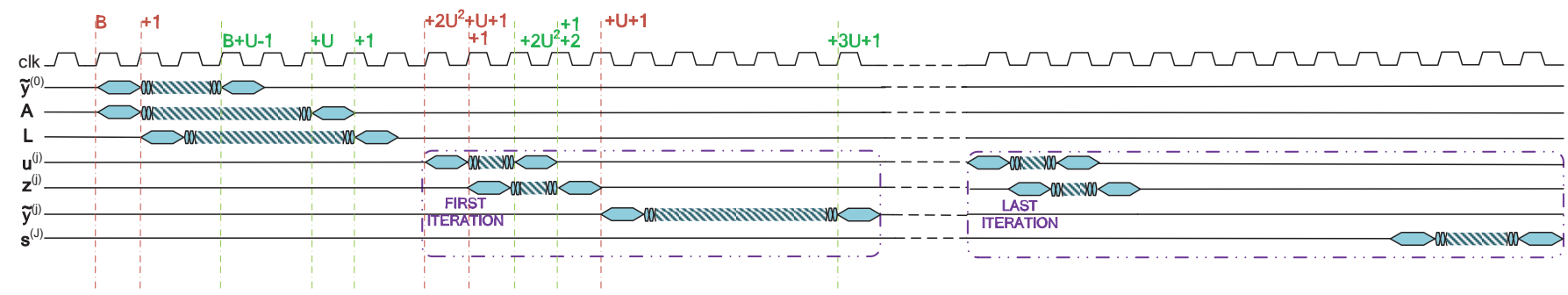

Fig. 13. Processing schedule of SSORSD detector.

TABLE III

FPGA RESUlTS WITH 128 BS ANTENNAS, 8 USERS, AND $\zeta_{B}=\zeta_{T}=0$.

\begin{tabular}{lllll}
\hline Detector & This Work & Z. Wu [ISCAS'16] [6] & B. Yin [ISCAS'15] [110] & Y. Xue [JSPS'18] [13] \\
\hline Algorithm & SSORSD $(J=2)$ & GS $(J=2)$ & CGLS $(J=3)$ & APSD $(J=2)$ \\
LUTs & $3,461(0.8 \%)$ & $18,976(4.38 \%)$ & $3,324(0.76 \%)$ & $2,947(0.68 \%)$ \\
FFs & $3,224(0.37 \%)$ & $15,864(1.83 \%)$ & $3,878(0.44 \%)$ & $3,697(0.42 \%)$ \\
DSP48s & $58(1.61 \%)$ & $232(6.44 \%)$ & $33(0.9 \%)$ & $50(1.39 \%)$ \\
BRAMs & $5(0.17 \%)$ & $6(0.2 \%)$ & $1(0.03 \%)$ & $6(0.2 \%)$ \\
\hline Latency [clocks] & 336 & 622 & 951 & 305 \\
Maximum frequency [MHz] & 245 & 309 & 412 & 199.942 \\
Throughput [Mbps] & 35 & 24 & 20 & 31.47 \\
\hline Throughput/LUTs & 10,112 & 1,265 & 6,017 & 10,679 \\
Throughput/FFs & 10,856 & 1,513 & 5,157 & 8,512 \\
\hline
\end{tabular}

by slightly revising the architecture. Other similar detectors can also employ this architecture.

\section{CONCLUSION}

In this paper, a universal pre-conditioned detector, SSORDS detector, is proposed for massive MIMO. Theoretical and numerical results have validated its advantages. Its universal implementation is also proposed. Future work will be directed towards its application in our 5G Cloud Platform.

\section{APPENDIX A \\ PRE-CONDitioned CDS Algorithms}

The pre-conditioned algorithms for DS detector, BB detector, and $\mathrm{CG}$ detector are given here.

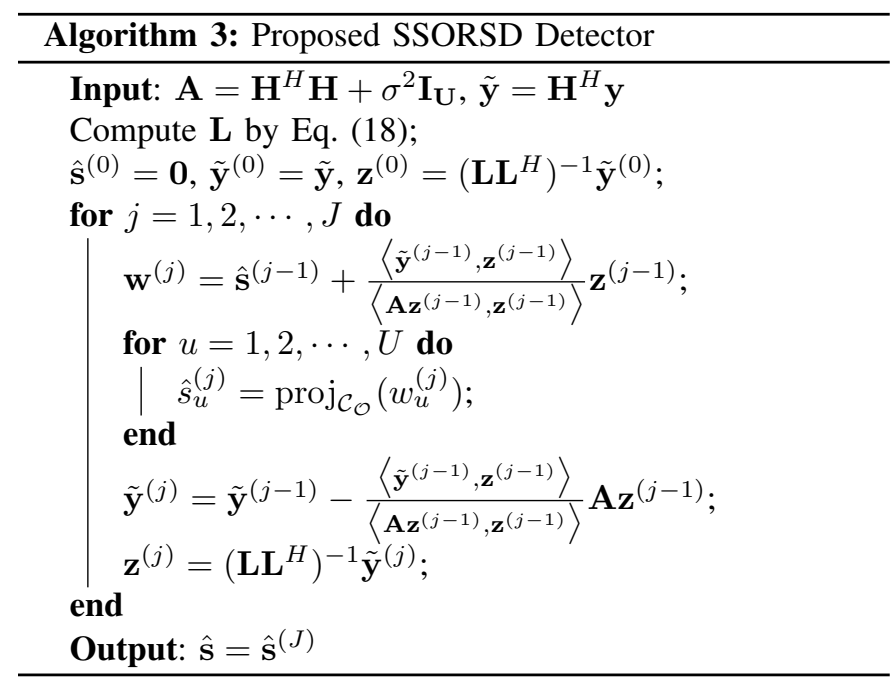

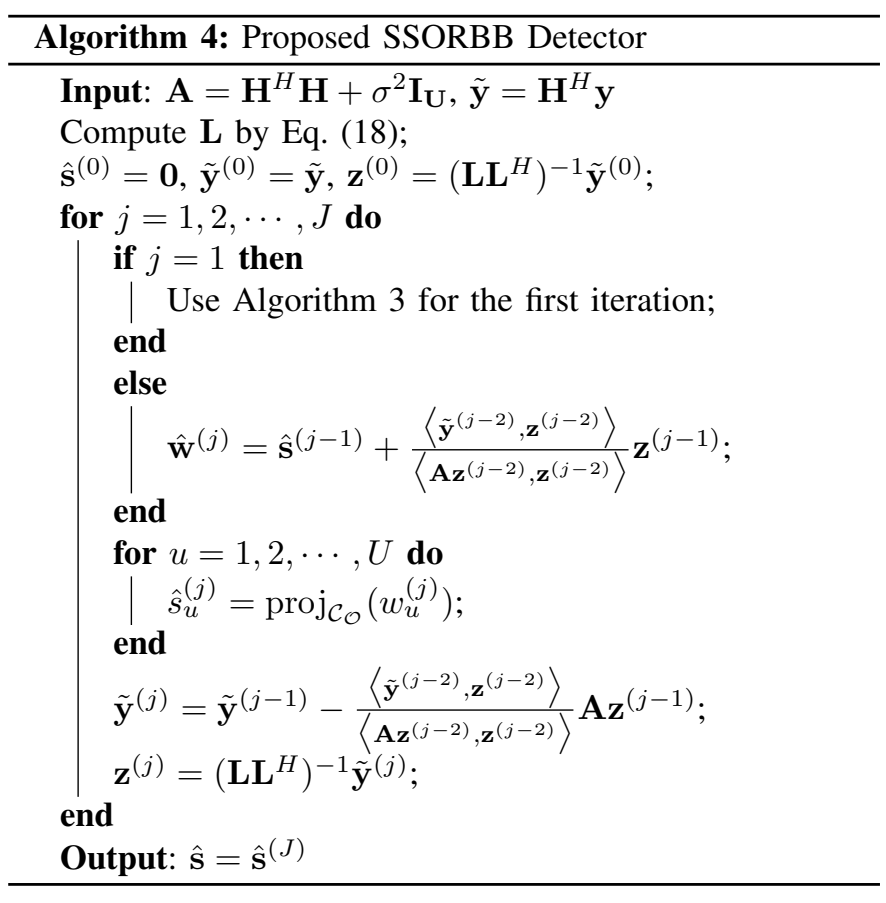

\section{APPENDIX B PROOF}

\section{A. Proof of Proposition $\square$}

Proof: Some preparations are required first.

Lemma. Since A is HPD, $\exists$ unitary matrix $\mathbf{U}$ such that

$$
\mathbf{A}=\mathbf{U}^{*} \boldsymbol{\Lambda} \mathbf{U}
$$

where $\boldsymbol{\Lambda}=\operatorname{diag}\left(\lambda_{1}(\mathbf{A}), \lambda_{2}(\mathbf{A}), \cdots, \lambda_{U}(\mathbf{A})\right)$ and $\lambda_{i}(\mathbf{A})(i=$ $1,2, \cdots, U) \in \mathbb{R}_{+}$.

Proof of this lemma can be referred to [30]. 


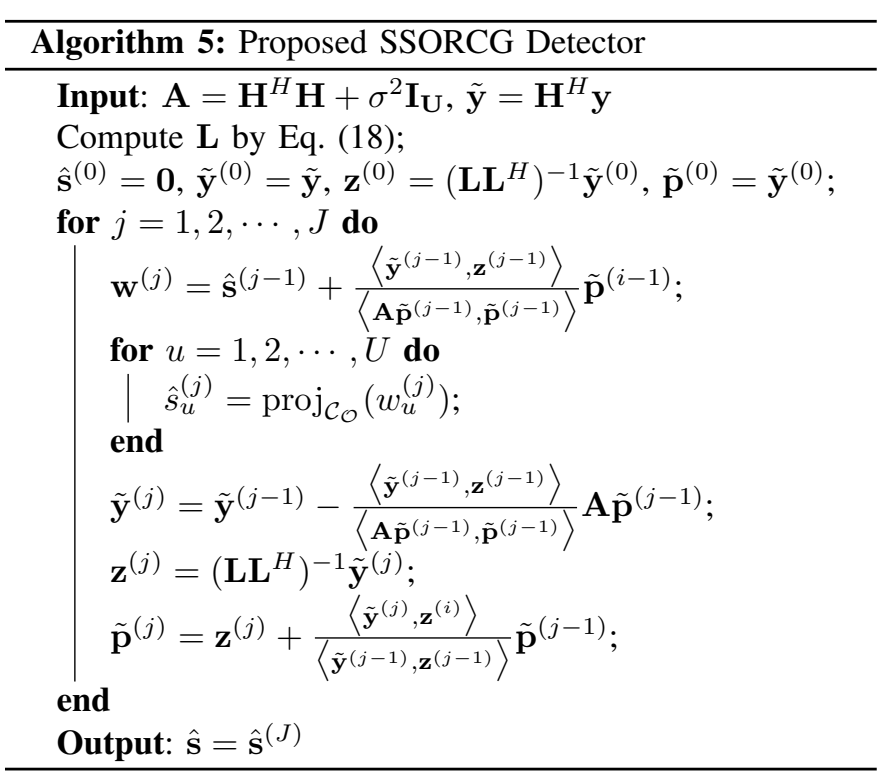

According to the definition of $\mathbf{A}$-norm, for $\forall \mathbf{u}, \mathbf{v} \in \mathbb{C}^{U}$ we have $\|\mathbf{u}+\mathbf{v}\|_{\mathbf{A}}^{2}-\|\mathbf{u}\|_{\mathbf{A}}^{2}=\|\mathbf{v}\|_{\mathbf{A}}^{2}+2 \Re(\langle\mathbf{A} \mathbf{v}, \mathbf{u}\rangle)$ and $\Re(\langle\mathbf{u}, \mathbf{v}\rangle)=\sum_{i=1}^{U} \Re\left(u_{i}\right) \Re\left(v_{i}\right)+\sum_{i=1}^{U} \Im\left(u_{i}\right) \Im\left(v_{i}\right)$.

Then the convergence difference between results with and without projection is as below:

$$
\begin{aligned}
& \left\|\hat{\mathbf{s}}^{(*)}-\hat{\mathbf{s}}_{1}^{(j)}\right\|_{\mathbf{A}}^{2}-\left\|\hat{\mathbf{s}}^{(*)}-\hat{\mathbf{s}}_{2}^{(j)}\right\|_{\mathbf{A}}^{2} \\
= & \left\|\hat{\mathbf{s}}_{2}^{(j)}-\hat{\mathbf{s}}_{1}^{(j)}\right\|_{\mathbf{A}}^{2}+2 \Re\left(\left\langle\mathbf{A}\left(\hat{\mathbf{s}}_{2}^{(j)}-\hat{\mathbf{s}}_{1}^{(j)}\right),\left(\hat{\mathbf{s}}^{(*)}-\hat{\mathbf{s}}_{2}^{(j)}\right)\right\rangle\right) \\
\geq & \lambda_{\min }\left\|\hat{\mathbf{s}}_{2}^{(j)}-\hat{\mathbf{s}}_{1}^{(j)}\right\|_{2}^{2}+2 \lambda_{\min } \Re\left(\left\langle\left(\hat{\mathbf{s}}_{2}^{(j)}-\hat{\mathbf{s}}_{1}^{(j)}\right),\left(\hat{\mathbf{s}}^{(*)}-\hat{\mathbf{s}}_{2}^{(j)}\right)\right\rangle\right) \\
\geq & \lambda_{\min }\left[\left\|\hat{\mathbf{s}}_{2}^{(j)}-\hat{\mathbf{s}}_{1}^{(j)}\right\|_{2}^{2}+2 \Re\left(\left\langle\left(\hat{\mathbf{s}}_{2}^{(j)}-\hat{\mathbf{s}}_{1}^{(j)}\right),\left(\hat{\mathbf{s}}^{(*)}-\hat{\mathbf{s}}_{2}^{(j)}\right)\right\rangle\right)\right],
\end{aligned}
$$

where $\lambda_{\min }$ is used to replace $\lambda_{\min }(\mathbf{A})$ for convenience.

Recalling the projective operation which pulls the result out of constellation set back to the margin of the original area, it is easy to deduce that both real and imaginary parts of each element of $\left(\hat{\mathbf{s}}_{2}^{(j)}-\hat{\mathbf{s}}_{1}^{(j)}\right)$ and $\left(\hat{\mathbf{s}}^{(*)}-\hat{\mathbf{s}}_{2}^{(j)}\right)$ are of the same sign or equal 0. Thus, $\Re\left(\left\langle\left(\hat{\mathbf{s}}_{2}^{(j)}-\hat{\mathbf{s}}_{1}^{(j)}\right),\left(\hat{\mathbf{s}}^{(*)}-\hat{\mathbf{s}}_{2}^{(j)}\right)\right\rangle\right) \geq 0$ always holds. Since $\lambda_{\min }$ and $\left\|\hat{\mathbf{s}}_{2}^{(j)}-\hat{\mathbf{s}}_{1}^{(j)}\right\|_{2}^{2}$ are also nonnegative, $\left\|\hat{\mathbf{S}}^{(*)}-\hat{\mathbf{s}}_{1}^{(j)}\right\|_{\mathbf{A}}^{2}-\left\|\hat{\mathbf{s}}^{(*)}-\hat{\mathbf{s}}_{2}^{(j)}\right\|_{\mathbf{A}}^{2} \geq 0$ is proved ultimately, verifying $\left\|\hat{\mathbf{s}}^{(*)}-\hat{\mathbf{s}}_{2}^{(j)}\right\|_{\mathbf{A}} \leq\left\|\hat{\mathbf{s}}^{(*)}-\hat{\mathbf{s}}_{1}^{(j)}\right\|_{\mathbf{A}}$.

\section{B. Proof of SCN Reduction for SSOR Pre-Conditioner}

Proof: Suppose $\mathbf{A}^{\prime}=\mathbf{L}^{-1} \mathbf{A} \mathbf{L}^{-H}$ is the matrix after preconditioning. The eigenvalue of $\mathbf{A}^{\prime}$ is:

$$
\begin{aligned}
\lambda_{i}\left(\mathbf{A}^{\prime}\right) & =\frac{\left\langle\mathbf{A}^{\prime} \mathbf{x}, \mathbf{x}\right\rangle}{\langle\mathbf{x}, \mathbf{x}\rangle}=\frac{\left\langle\mathbf{L}^{-1} \mathbf{A} \mathbf{L}^{-H} \mathbf{x}, \mathbf{x}\right\rangle}{\langle\mathbf{x}, \mathbf{x}\rangle} \\
& =\frac{\left\langle\mathbf{A} \mathbf{L}^{-H} \mathbf{x}, \mathbf{L}^{-H} \mathbf{x}\right\rangle}{<\mathbf{x}, \mathbf{x}\rangle} \\
& =\frac{\langle\mathbf{A y}, \mathbf{y}\rangle}{\left\langle\mathbf{L}^{H} \mathbf{y}, \mathbf{L}^{H} \mathbf{y}\right\rangle}=\frac{\langle\mathbf{A y}, \mathbf{y}\rangle}{\langle\mathbf{M y}, \mathbf{y}\rangle}
\end{aligned}
$$

where $\mathbf{y}=\mathbf{L}^{-H} \mathbf{x}$. According to the expression of $\mathbf{M}$ we have

$$
\mathbf{M}=\frac{\mathbf{D}-\omega\left(\mathbf{C}_{\mathbf{L}}+\mathbf{C}_{\mathbf{L}}{ }^{H}\right)+\omega^{2} \mathbf{C}_{\mathbf{L}} \mathbf{D}^{-1} \mathbf{C}_{\mathbf{L}}{ }^{H}}{\omega(2-\omega)}
$$

$$
=\mathbf{A}+\frac{\left[(\omega-1) \mathbf{D}-\omega \mathbf{C}_{\mathbf{L}}\right] \mathbf{D}^{-1}\left[(\omega-1) \mathbf{D}-\omega \mathbf{C}_{\mathbf{L}}{ }^{H}\right]}{\omega(2-\omega)} .
$$

Thus,

$$
\begin{aligned}
<\mathbf{M y}, \mathbf{y}> & =<\mathbf{A y}, \mathbf{y}>+\frac{1}{\omega(2-\omega)}<\mathbf{D}^{-\frac{1}{2}}[(\omega-1) \mathbf{D} \\
& \left.-\omega \mathbf{C}_{\mathbf{L}}\right] \mathbf{y}, \mathbf{D}^{-\frac{1}{2}}\left[(\omega-1) \mathbf{D}-\omega \mathbf{C}_{\mathbf{L}}{ }^{H}\right] \mathbf{y}> \\
& \geq<\mathbf{A y}, \mathbf{y}>\text {, when } \mathbf{y} \neq 0,0<\omega<2 .
\end{aligned}
$$

In view of the definition of eigenvalue, we have $\lambda_{i}\left(\mathbf{A}^{\prime}\right) \leq$ 1, i.e., $\lambda_{\max }\left(\mathbf{A}^{\prime}\right) \leq 1$.

Then consider the smallest eigenvalue $\lambda_{\min }\left(\mathbf{A}^{\prime}\right)$. $\mathbf{M}$ can be rewritten as:

$$
\begin{aligned}
\mathbf{M} & =\frac{1}{2-\omega}\left\{\frac{1}{\omega} \mathbf{D}-\left(\mathbf{C}_{\mathbf{L}}+\mathbf{C}_{\mathbf{L}}{ }^{H}\right)+\omega \mathbf{C}_{\mathbf{L}} \mathbf{D}^{-1} \mathbf{C}_{\mathbf{L}}{ }^{H}\right\} \\
& =\frac{1}{2-\omega}\left\{\mathbf{A}+\frac{(2-\omega)^{2}}{4 \omega} \mathbf{D}+\omega\left(\mathbf{C}_{\mathbf{L}} \mathbf{D}^{-1} \mathbf{C}_{\mathbf{L}}{ }^{H}-\frac{1}{4} \mathbf{D}\right)\right\} .
\end{aligned}
$$

Therefore,

$$
\begin{aligned}
\lambda_{i}\left(\mathbf{A}^{\prime}\right) & =\left(\frac { 1 } { 2 - \omega } \left\{1+\frac{(2-\omega)^{2}<\mathbf{D} \mathbf{y}, \mathbf{y}>}{4 \omega<\mathbf{A y}, \mathbf{y}>}\right.\right. \\
& \left.\left.+\omega \frac{<\left(\mathbf{C}_{\mathbf{L}} \mathbf{D}^{-1} \mathbf{C}_{\mathbf{L}}{ }^{H}-\frac{1}{4} \mathbf{D}\right) \mathbf{y}, \mathbf{y}>}{<\mathbf{A y}, \mathbf{y}>}\right\}\right)^{-1} .
\end{aligned}
$$

If the following relationship holds (in fact it can be verified here):

$$
<\left(\mathbf{C}_{\mathbf{L}} \mathbf{D}^{-1} \mathbf{C}_{\mathbf{L}}{ }^{H}-\frac{1}{4} \mathbf{D}\right) \mathbf{y}, \mathbf{y}>\leq 0, \forall \mathbf{y} \neq 0,
$$

we have

$$
\begin{aligned}
\lambda_{\min }\left(\mathbf{A}^{\prime}\right) & =\min _{\mathbf{y} \neq 0} K \\
& \geq\left\{\frac{1}{2-\omega}\left[1+\frac{(2-\omega)^{2}}{4 \omega} \mu\right]\right\}^{-1}=\frac{1}{s},
\end{aligned}
$$

where $\mu=\max _{\mathbf{y} \neq 0} \frac{\langle\mathbf{D y}, \mathbf{y}\rangle}{\langle\mathbf{A y}, \mathbf{y}\rangle}, s=\frac{1}{2-\omega}+\frac{2-\omega}{4 \omega} \mu$.

Let $\frac{\partial s}{\partial \omega}=0$ we have

$$
\omega=2 /(1+\sqrt{2 / \mu}) .
$$

Meanwhile, $s$ attains its smallest value:

$$
s_{\min }=\frac{1}{2}(1+\sqrt{2 \mu}) .
$$

For this $\omega$, we have

$$
\begin{aligned}
\kappa^{\prime} & =\frac{\lambda_{\max }\left(\mathbf{A}^{\prime}\right)}{\lambda_{\min }\left(\mathbf{A}^{\prime}\right)} \\
& \leq\left\{\frac{1}{\frac{1}{2}(1+\sqrt{2 \mu})}\right\}^{-1} \\
& =\frac{1}{2}(1+\sqrt{2 \mu}) .
\end{aligned}
$$

As for the expression of $\mu$ :

$$
\begin{aligned}
\mu & =\max _{\mathbf{y} \neq 0} \frac{\langle\mathbf{D} \mathbf{y}, \mathbf{y}\rangle}{\langle\mathbf{A y}, \mathbf{y}\rangle}=\max _{\mathbf{y} \neq 0} \frac{\left\langle\mathbf{D}^{\frac{1}{2}} \mathbf{y}, \mathbf{D}^{\frac{1}{2}} \mathbf{y}\right\rangle}{\langle\mathbf{A y}, \mathbf{y}\rangle} \\
& =\max _{\mathbf{z} \neq 0} \frac{<\mathbf{z}, \mathbf{z}\rangle}{\left\langle\mathbf{A D}^{-\frac{1}{2}} \mathbf{z}, \mathbf{D}^{-\frac{1}{2}} \mathbf{z}\right\rangle}=\frac{1}{p^{*}},
\end{aligned}
$$


and

$$
\begin{aligned}
p^{*} & =\min _{\mathbf{z} \neq 0} \frac{\left\langle\mathbf{D}^{-\frac{1}{2}} \mathbf{A D}^{-\frac{1}{2}} \mathbf{z}, \mathbf{z}\right\rangle}{\langle\mathbf{z}, \mathbf{z}>} \\
& =\lambda_{\min }\left(\mathbf{D}^{-\frac{1}{2}} \mathbf{A D}^{-\frac{1}{2}}\right) \\
& =\lambda_{\min }\left(\mathbf{D}^{-1} \mathbf{A}\right)=\lambda_{\min }\left(1-\mathbf{D}^{-1} \mathbf{C}\right) \\
& =1-\max _{i} \lambda_{i}(\mathbf{J}),
\end{aligned}
$$

where $\mathbf{z}=\mathbf{D}^{\frac{1}{2}} \mathbf{y}, \mathbf{C}=\mathbf{D}-\mathbf{A}$ and $\mathbf{J}=\mathbf{D}^{-1} \mathbf{C}$. For the problem presented here, it can be proved [31] that

$$
\max _{i} \lambda_{i}(\mathbf{J})=1-O\left(h^{2}\right),
$$

where $h$ equals $\frac{1}{U+1}$, and $O\left(h^{2}\right)$ is an infinitesimal as the same order as $h$.

As a result, $\mu=O\left(h^{-2}\right)$ and $\kappa^{\prime} \leq O\left(h^{-1}\right)$. On the other hand, the SCN of the original matrix $\mathbf{A}$ is $\kappa=O\left(h^{-2}\right)$ [32]. In the end, the new SCN approximately equals the square root of the original one.

\section{REFERENCES}

[1] F. Boccardi, R. W. Heath, A. Lozano et al., "Five disruptive technology directions for 5G," IEEE Commun. Mag., vol. 52, no. 2, pp. 74-80, February 2014.

[2] D. Micciancio, "The hardness of the closest vector problem with preprocessing," IEEE Trans. Inf. Theory, vol. 47, no. 3, pp. 1212-1215, Mar 2001.

[3] H. Q. Ngo, M. Matthaiou, T. Q. Duong et al., "Uplink performance analysis of multicell MU-SIMO systems with ZF receivers," IEEE Trans. Veh. Technol., vol. 62, no. 9, pp. 4471-4483, 2012.

[4] Y. C. Liang, G. Pan, and Z. D. Bai, "Asymptotic performance of MMSE receivers for large systems using random matrix theory," IEEE Trans. Inf. Theory, vol. 53, no. 11, pp. 4173-4190, 2007.

[5] L. N. Trefethen and D. Bau III, Numerical linear algebra. Siam, 1997, vol. 50.

[6] Z. Wu, C. Zhang, Y. Xue et al., "Efficient architecture for soft-output massive MIMO detection with Gauss-Seidel method," in Proc. IEEE Intl. Symp. on Cir. and Syst. (ISCAS), 2016, pp. 1886-1889.

[7] M. Avriel, Nonlinear programming: analysis and methods. Courier Corporation, 2003.

[8] R. Fletcher, "On the barzilai-borwein method," in Optim. and Control with Appl. Springer, 2005, pp. 235-256.

[9] M. R. Hestenes and E. Stiefel, Methods of conjugate gradients for solving linear systems. NBS Washington, DC, 1952, vol. 49, no. 1.

[10] B. Yin, M. Wu, J. R. Cavallaro et al., "VLSI design of large-scale softoutput MIMO detection using conjugate gradients," in Proc. IEEE Intl. Symp. on Cir. and Syst. (ISCAS), 2015, pp. 1498-1501.

[11] Y. Xue, C. Zhang, S. Zhang et al., "Steepest descent method based soft-output detection for massive MIMO uplink," in Proc. IEEE Intl. Workshop on Signal Process. Syst. (SiPS), 2016, pp. 273-278.

[12] J. Jin, Y. Xue, Y.-L. Ueng et al., "A split pre-conditioned conjugate gradient method for massive MIMO detection," in Proc. IEEE Intl. Workshop on Signal Process. Syst. (SiPS),, 2017, pp. 1-6.

[13] Y. Xue, Z. Wu, J. Yang et al., "Adaptive Preconditioned Iterative Linear Detection and Architecture for Massive MU-MIMO Uplink," J. Signal Process. Syst., pp. 1-15, 2017.

[14] B. E. Godana and T. Ekman, "Parametrization based limited feedback design for correlated MIMO channels using new statistical models," IEEE Trans. Wireless Commun., vol. 12, no. 10, pp. 5172-5184, 2013.

[15] A. Paulraj, R. Nabar, and D. Gore, Introduction to space-time wireless communications. Cambridge university press, 2003.

[16] P. H. Tan, L. K. Rasmussen, and T. J. Lim, "Constrained maximumlikelihood detection in CDMA," IEEE Trans. Commun., vol. 49, no. 1, pp. 142-153, 2001

[17] A. Yener, R. D. Yates, and S. Ulukus, "Cdma multiuser detection: A nonlinear programming approach," IEEE Trans. Commun., vol. 50, no. 6, pp. 1016-1024, 2002.

[18] C. Thrampoulidis, E. Abbasi, W. Xu et al., "Ber analysis of the box relaxation for BPSK signal recovery," in Proc. IEEE Int. Conf. on Acoust., Speech and Signal Process. (ICASSP), 2016, pp. 3776-3780.
[19] M. Wu, C. Dick, J. R. Cavallaro et al., "High-throughput data detection for massive MU-MIMO-OFDM using coordinate descent," IEEE Trans. Circuits Syst. I, Reg. Papers, vol. 63, no. 12, pp. 2357-2367, 2016.

[20] M. D. Pore, "Quadratic programming with linear inequality constraints," Ph.D. dissertation, Texas Tech University, 1969.

[21] Y. Saad, Iterative methods for sparse linear systems. SIAM, 2003.

[22] G. Strang, "Linear algebra and its applications," Math. of Comp., vol. 435, no. 8, p. 175C192, 1988.

[23] A. van der Sluis and H. van der Vorst, "The rate of convergence of Conjugate Gradients," Numer. Math., vol. 48, no. 5, pp. 543-560, 1986.

[24] M. Matthaiou, M. R. Mckay, P. J. Smith et al., "On the condition number distribution of complex wishart matrices," IEEE Trans. Commun., vol. 58, no. 6, pp. 1705-1717, 2010.

[25] G. J. Habetler and E. L. Wachspress, "Symmetric successive overrelaxation in solving diffusion difference equations," Math. of Comp., pp. 356-362, 1961.

[26] K. K. Parhi, VLSI digital signal processing systems: design and implementation. John Wiley \& Sons, 2007.

[27] N. Petkov, "Systolic parallel processing (Advances in Parallel Computing, Vol 5)," North-Holland, 1993.

[28] F. Gaston and G. Irwin, "Systolic Kalman filtering: an overview," in Proc. IEE, vol. 137, no. 4, 1990, pp. 235-244.

[29] M. Wu, B. Yin, A. Vosoughi et al., "Approximate matrix inversion for high-throughput data detection in the large-scale MIMO uplink," in Proc. IEEE Int. Symp. on Cir. and Syst. (ISCAS), 2013, pp. 2155-2158.

[30] J. H. Wilkinson, The algebraic eigenvalue problem. Clarendon Press Oxford, 1965, vol. 87.

[31] H. Jiagan, The Iterative Computing Method of Linear Algebra Equations. Science Press, 1991.

[32] R. Beauwens, "Iterative solution methods," Appl. Num. Math., vol. 51, no. 4, pp. 437-450, 2004. 